Revue d'histoire de l'Amérique française

REVUE D.HISTOIRE DE L'AMÉRIQUE FRANÇAISE

\title{
Liste des engagés pour le Canada au XVIIe siècle (1634-1715)
}

\section{G. Debien}

Volume 6, numéro 3, décembre 1952

URI : https://id.erudit.org/iderudit/301535ar

DOI : https://doi.org/10.7202/301535ar

Aller au sommaire du numéro

Éditeur(s)

Institut d'histoire de l'Amérique française

ISSN

0035-2357 (imprimé)

1492-1383 (numérique)

Découvrir la revue

Citer cet article

Debien, G. (1952). Liste des engagés pour le Canada au XVIIe siècle (1634-1715).

Revue d'histoire de l'Amérique française, 6(3), 374-407.

https://doi.org/10.7202/301535ar d'utilisation que vous pouvez consulter en ligne.

https://apropos.erudit.org/fr/usagers/politique-dutilisation/ 


\section{LISTE DES ENGAGÉS POUR LE CANADA AU XVIIE SIECLE}

\section{(1634-1715)}

Cette liste fait suite à l'article de M. G. Debien, Le Caire, Egypte, paru dans notre numéro de septembre dernier aux pages 177-220. C'est aussi une seconde tranche de cette liste. Voir le début, VI, no 2: 221-233.

1644

a André Tuffet... (Teuleron not. Reg.).

[ 178-180] 8 janvier Robert ConMIER, maitre charpentier de La Rochelle et Marie PÉraud sa femme, Thomas Cormier, leur fils, partant sur le Petit-SaintPierre, dont est maître Barbau, pour aller travailler pour les sieurs Tuffet, Duchanin et Deschervery, de son métier de charpentier aux ordres du sieur Louis Tuffet, commandant le fort SaintPierre. 120 l. par an à eux tous. (fol. $165 \mathrm{v}^{\circ}$ ).

(en marge: ledit Cormier pourra emmener son fils Jean âgé de 20 mois).

[ 181 ] Jean de la Trourtte, tireur de scie, demeurant à La Rochelle pour travailler de son métier, 2 ans, 80 livres par an, 40 d'avance, signe; (fol. 166).
[ 182 ] 15 février - Nicolas LETARD' marinier, de La Rochelle, $105 \mathrm{l}$. par an, la moitié d'avance, signe (fol. $188 \mathrm{v}^{\circ}$ ).

[183] Mathurin Hera, id., des Sables ${ }^{138}$, pour 2 ans, id.

[184 ] Pierre Servant, id. de La Rochelle, 2 ans, 80 l. par an, la moitié d'avance (fol. 189).

[185] Guillaume Brunetier, id. id. 2 ans, 105 l. par an, dont la moitié d'avance (fol. $189 \mathrm{v}^{\circ}$ ).

[ 186 ] 26 février - Pierre Dubors, charpentier, de Marennes, 2 ans, 200 1. par an, 60 l. d'avance, signe; (fol. 197).

[ 187 ] 26 février - Jean FapJot, dit Le Rousseau, de La Rochelle, pour 2 ans comme tireur de scie, 80 l. par an, 40 d'avance (fol. 197).

[ 188-189] 30 mars - Adam DeLGAOURDI, marchand, de SaintJean de Luz ${ }^{139}$ s'engage avec Joannis, son fils et Laurent de Harabillagne, mariniers, qui

138. (Vendée).

139. Chef-lieu de canton, arrondissement de Bayonne (Basses-Pyrénées). 
signent, le père pour faire la troque, les mariniers pour naviguer. 1 an, et ils auront les deux tiers des profits (fol. $221 \mathrm{v}^{\circ}$ ).

a Antoine Cheffault, pour la Compagnie générale de la NouvelleFrance à Québec, pour 3 ans, aller et retour payés (Teuleron, not. Reg.).

[ 191 ] 4 avril - Jean Roy, soldat et matelot, de Villedieu en Breta$\mathrm{gne}^{140} 80$ l. par an, 40 d'avance (fol. $228 \mathrm{v}^{\circ}$ ).

[192] Francois Pilorecq, id. id. mêmes gages (fol. 229).

[ 193 ] Jean LedenicQ, id. id. id. (fol. $229 \mathrm{v}^{\circ}$ ).

[ 194 ] Antoine Catre, id. de SaintNazaire ${ }^{111}$, 100 l. dont 601 . d'avance. (fol. 230).

[ 195 ] Jean Coussant, soldat, de La Rochelle, 90 1. par an, dont 50 d'avance (fol. $230 \mathrm{v}^{\circ}$ ).

[196] François BARY, soldat, d'Angers, 75 1. dont 45 d'avance. (fol. 231).

[ 197 ] Jean Constantin, soldat et laboureur, de Saint-Jean d'Angély, 75 liv. par an dont 40 d'av. (fol. $231 \mathrm{v}^{\circ}$ ).

[ 198 ] Gédéon Coindet, de SaintDenis d'Oleron ${ }^{142}, 120$ l. par an, 80 d'avance (fol. 232).

[ 199 ] Jean GuIllot, charpentier, id. 200 l. par an, dont 100 d'av. (fol. $232 \mathrm{v}^{\circ}$ ).
[ 200 ] Charles Chevalier, soldat, de Pontoise ${ }^{13}, 75$ 1. par an, 25 d'av. signe (fol. 233).

[201] 9 avril - Etienne ChevaLIER, id. de Saint-Aupert ${ }^{144}, 751$. par an 75 l. d'avance. Signe (id.). [202] 5 avril - Nicolas Colleson, soldat et aide-chirurgien, de $\mathrm{Pa}$ ris, 75 l. par an, 75 l. d'avance. Signe (fol. $233 \mathrm{v}^{\circ}$ ).

[203] 5 avril - Pierre Mox, matelot et soldat, de Marennes ${ }^{145}$, 105 1. par an, 60 d'avance (fol. 234).

[204] 6 avril - Jean Miceleau, soldat de Nantes, 90 l. par an, dont 60 d'av. (fol. $235 \mathrm{v}^{\circ}$ ).

[ 205 ] 8 avril - Mathurin LEBRET, soldat, de Rouen, id. (fol. $240 \mathrm{v}^{\circ}$ ). [206 ] Gaspard Pontiel, dit des Rangs, du Havre, soldat, id. (fol. 241).

A Pierre Prévost, marchand, faisant pour la Compagnie de la Nouvelle-France, pour 3 ans, aller et retour, (Teuleron, not. Reg.).

[207] 10 avril - Jean Noues, laboureur, de La Rochelle, 751. par an, dont 30 d'av. (fol. 249).

[208 120 avril - Jacques LévesQUE, id. id. id. (fol. 254).

[209] 30 avril - Jean BARILLET, charpentier, id. 100 l. par an, 40 d'av. (fol. 269).

[210] Jacques Richard, lab. id.

140. Ne serait-ce pas l'île d'Yeu?

141. Chef-lieu d'arrondissement de la Loire-Inférieure.

142. Commune du canton de Saint-Pierre d'Oleron (Charente-Maritime).

143. Chef-lieu de canton de la Seine-et-Oise.

144. Saint-Aupre (Isère)? ou Saint-Aubert Nord)?, ou Saint-Aubert-surOrne (Orne)?

145. (Charente-Maritime). 
id. 75 l. par an, 30 d'av. (fol. [217] Nicolas Leroy, id. id. 60 l. $\left.269 \mathrm{v}^{\circ}\right)$.

[211] 2 mai - Jean Doyon, id., au bourg d'Esnandes en Aunis ${ }^{146^{\prime}}$ 60 l. par an, 30 d'av. (fol. $270 \mathrm{v}^{\circ}$ )',

"A Hiérosme Le Royer, sieur de la Dauversière, procureur de la Compagnie de MM. les Associés de la Conversion des Sauvages de la Nouvelle France en l'île de Montréal, demeurant ordinairement à la Flesche, étant de présent logé en cette ville en la maison de $\mathrm{s}^{\mathrm{r}}$ Jacques Mousnier, marchand" s'engagent pour 3 ans (Teuleron, not. Reg.).

[212 20 avril - Simon Barraut, tonnelier, né au bourg de Marsé $e^{147}$ en Aunis, à 601 . par an, signe (fol. $253 \mathrm{v}^{\circ}$ ).

[ 213 ] 20 avril - Anthoine Vedet, cloutier, de Clermont en Auver$\mathrm{gne}^{148}, 90$ l. par an (fol. 253 bis).

[ 214 ] François Lardereau, matelot canonnier, de Brouage, $100 \mathrm{l}$. par an. (fol. 253 bis).

[215] Mathurin Lemousnier, bêcheur, de Clermont près la Flèche ${ }^{149}$, pour 5 ans, à $70 \mathrm{l}$. par an. Signe (fol. 255).

[216] Jean VAYDIÉ, id. de SaintGermain près la Flèche $e^{150}$, pour 5 ans, mêmes gages. (id.). par an.

[218 ] Mathurin Boudefeu, id. d'Igé, pays du Perche ${ }^{151}$, pour 5 ans à $60 \mathrm{l}$. par an (fol. 255).

[219] Jean Leduc, id. id.

[220] Paul Chambor, id. de Feucombeaj près Blanc en Berry ${ }^{152}$ pour 3 ans, à 75 l. par an (fol. 256). [ 221 ] Julien Vautabon, chaudronnier, de La Rochelle, pour 3 ans à 80 l. par an (fol. $254 \mathrm{v}^{\circ}$ ).

[ 222 ] Christophe Leflot, marinier, de Lanerie (?) en Bretagne ${ }^{153}$ pour 3 ans à 75 l. (fol. 261).

[223] Jean Fouchereau, matelot, de Brouage, 3 ans, 40 l. par an. Signe. (fol. 265).

[ 224 ] Bernard Lardereau, maître maçon, id. id. 120 l. par an. (fol. 267).

[225-226 ] 29 avril - Anthoine Le Bouesme, armurier, id. etson fils Louis, à 120 l. par an (fol. 268).

[227] 2 mai - Simon Richome, charron et charpentier, de Brouage, pour 3 ans, à $100 \mathrm{l}$. par an. Signe (fol. 268).

[ 228 ] 5 mai - Jean Gourraguer, matelot d'Audiarne en Bretagne ${ }^{154}, 3$ ans, à $60 \mathrm{l}$. par an (fol. 272).

[ 229 ] Simon Moisne, lab. de Mar-

146. Commune du canton Ouest et de l'arrondissement de La Rochelle.

147. Ou Marsay, c'est-a-dire Marsilly, chef-lieu de canton de l'arrondissement de La Rochelle.

148. Clermont-Ferrand (Puy-de-Dôme).

149. Commune du canton de La Flèche (Sarthe).

150. Saint-Germain d'Arcé, commune du canton de Lude (Sarthe).

151. Commune du canton de Bellême (Orne).

152. Fontgombault, près du Blanc, canton de Tournon Saint-Martin (Indre).

153. Non identifié.

154. Pour Audierne, commune du canton de Pont-Croix, Finistère. 
saye en Aunis ${ }^{155}, 3$ ans, 501. (fol. $270 \mathrm{v}^{\circ}$ ).

[ 230 ] Pierre Mousnier, matelot, du Château d'Oleron ${ }^{156}$, id. $80 \mathrm{l}$. Signe (fol. 272).

[ 231 ] Blaise Jeuillet ${ }^{157}$, bêcheur, 3 ans, 70 l. par an. (fol. $257 \mathrm{v}^{\circ}$ ). [ 232 ] Jacques Regnaud, id. de la Rochelle, 60 l. par an (fol. $257 \mathrm{v}^{\circ}$ ).

\section{5}

à Emmanuel Le Borgne, de La Rochelle, "pour lui et M. le gouverneur de la Nouvelle-France" pour travailler à la coupe des bois et à faire des soliveaux et autres choses, pour 3 ans, nourriture et aller et retour payés. Le Borgne se réservant les deux tiers des bois coupés, les ouvriers se partageant l'autre tiers, $60 \mathrm{l}$. données en avances (Teuleron, not. Reg. fol. 35):

[233] 5 avril - Nicolas Pinel, charpentier de marine, de La Rochelle.

[ 234 ] Louis BlaNCHARD, charpentier de marine, de La Rochelle.

[ 235 ] Jean Perrin, charpentier de marine, de La Rochelle.

[ 236 ] Pierre JANviere ${ }^{158}$, charpentier de marine, de La Rochelle.

[ 237 ] Jean ChaILLot, charpentier de marine, de La Rochelle.

à Pierre Le Gardeur écuyer, sieur de Repentigny, "faisant tant pour lui que pour les habitants dud. pays", pour 3 ans, aller et retour payés (Teuleron, not).

[238] 10 juin - Pierre MarcheTEAU, ${ }^{159}$ maçon de La Rochelle, 64 l. par an, 44 l. d'avance.

au même comme directeur des embarquements de la Nouvelle-France, faisant pour lui et pour Mme de la Pelletrie, demeurant à Québec, 3 ans, aller et retour payés:

[239] 10 juin - Bernard PICHON, maître maçon, tailleur de pierre à La Rochelle, 135 l. par an, 75 l. et 17 l. d'avance (fol. 67).

[240] Louis Jouet, maître charpentier, de Ia Rochelle, $135 \mathrm{l}$. par an; avances $70 \mathrm{l}$. et $71 \mathrm{l}$. (fol. $67 \mathrm{v}^{\circ}$ ).

[241] Jean Nepveu, maçon, de Saint-Georges de Montaigu, en Poitou $^{160}, 60$ l. par an.

[242] 16 juin - A Pierre LE GarDEUR, pour les habitants de Québec, Jean Groussain, laboureur, "demeurant en cette ville". (Teuleron, not. liasse).

\section{6}

à Nicolas Denis, marchand à La Rochelle, pour 3 ans au Canada. [243] 19 mars - André LAGoRT, saulnier, de l'île de Ré, et Etienne, son fils, 75 l. par an; al-

155. La finale y ou é étant indifférente au XVIIe siècle, il faut lire Marsilly.

156. Chef-lieu de canton, de la Charente-Maritime.

157. Ou Juillet.

158. On peut aussi bien lire JAVIERE.

159. Dans l'acte, il y a MARSEREAU, en marge: MARCHETEAU.

160. Commune du canton de Montaigu, arrondissement, de La Roche-sur-Yon (Vendée). 
ler et retour. (Teuleron, not. Reg. fol. 191).

à Pierre Le Gardeur "faisant pour les habitants de la NouvelleFrance".

[244] 1er mai - Jean Doucet, maçon, tailleur de pierre, d'Ars en l'île de Ré pour 3 ans, à $100 \mathrm{l}$. par an, 60 l. d'avance, aller et retour payés. Signe. (Teuleron, not. reg.).

à Emmanuel Le Borgne, marchand banquier à La Rochelle, faisant pour le go iverneur de l'Acadie, s'engagent à aller à Port-Royal, côte de l'Acadie, pour 2 ans, à 160 1. par an, 50 d'avance, aller et retour payés. "S'ils travaillent pour d'autres personnes, leur salaire leur appartiendra".

[ 245 ] 10 juin - Barthélémy ChasTEAU, armurier, de Mirebeau ${ }^{161}$.

[245] Jean Lamaison, armurier, de Lectoure en Gascogne ${ }^{162}$ (Teuleron, not.).

\section{7}

a Noël Juchereau $\mathrm{s}^{\mathbf{r}} \mathrm{du}$ Chastellier, procureur général des habitants de la Nouvelle France, pour 3 ans (Teuleron, not. Reg.).

[ 247 ] 9 mars - Mathurin TRUT, laboureur, de Cognac ${ }^{163}$, 65 l. par an, aller et retour payés. Signe. (fol. $41 \mathrm{v}^{\circ}$ ).

[248] 1er avril - Adam DeL-
zAUDY, capitaine de navire, de Saint-Jear de Luz, de présent à La Rochelle, aller et retour, 200 1. par an, 200 livres d'avance. Signe (fol. 50).

[ 247 ] 5 mai - Jean Margat, scieur de long, de Sigogne, paroisse du Thou, en Aunis ${ }^{164}, 60$ 1. par an. 30 d'avance.

[ 250 ] Jacques Drouet, de la Jarne, scieur de long, mêmes conditions.

au P. Quantin, jésuite, engagements par devant Teuleron (Liasse): [251-252 ] 12 avril - Pierre PorCHET, laboureur à bœufs, demeurant à La Rochelle et de Martine Duruau, sa femme, comme métayers d'une des métairies de la Compagnie à Québec.

[253-254] 12 avril - Jacques BADEAU, laboureur à bœufs et Jeanne Ardouin, sa femme mêmes conditions, comme métayers (Teuleron, Reg. fol. 55).

à Emmanuel Le Borgne, marchand à La Rochelle, faisant pour $M$. Charles de Menou, chevalier, gouverneur et lieutenant-général pour le roi au pays de NouvelleFrance, pour 2 ans, aller et retour payés, 150 l. par an.

[ 255 ] 16 avril - Gilles De Coster, menuisier, de Saint-Nicolas près d'Anvers. Signe (Teuleron, not fol. $57 \mathrm{v}^{\circ}$ ).

à Augustin Hébert dit Jolycœur, habitant de Montréal.

161. Chef-lieu de canton de la Vienne.

162. Chef-lieu d'arrondissement du Gers.

163. Chef-lieu d'arrondissement de la Charente.

164. Le Thou, commune du canton d'Aigrefeuille, arrondissement de Rochefort (Charente-Maritime). 
[256] 2 mai - René Pigneau, charpentier de gros œuvre, de La Roche-sur-Yon en Poitou, pour 5 ans, à 75 l. par an, entretien et aller et retour payés. (Teuleron, not. Reg. fol. $65 \mathrm{v}^{\circ}$ ).

\section{8}

à Jacques Maheut, habitant de la Nouvelle-France, pour 3 ans, à 60 livres par an, 40 l. d'avance; aller et retour payés (Teuleron, not. liasse).

[257] 12 mai - Pierre Landais, laboureur, demeurant à La Rochelle, "pour labourer la terre".

à Jean Humeau, habitant de Québec, pour 3 ans, à 60 l. par an. [ 259 ] 12 mai - Thomas Charroux tessier, de Bonnétable, au Perche $^{165}$ (Teuleron, not. liasse).

[259b] 7 juillet - Engagement de Bertrand de Leglebat, bonnetier à Toulouse, pour 3 ans à 80 livres par an, à Pierre Gagneur qui agit au nom de la Compagnie des Indes Occidentales. Leglebat ira au Canada. Il signe. (Teuleron, not.)

1649

au P. Jean Lrégeors, jésuite, pour le $\mathrm{s}^{\mathrm{r}}$ Pierre Boucher, des TroisRivières.
[260] 16 mars - Nicolas Petit, laboureur, du Gué d'Alleré ${ }^{166}$.

au sieur Noël Morın, habitant de Québec, par l'intermédiaire de Noël Belanger, conseiller $d u$ roi (Teuleron, not. liasse).

[ 261 ] 4 juin - Pierre Paillereau, laboureur, de Villedoux ${ }^{167}$.

1651

à honorable homme Jean BourDON, habitant de Québec, s'engagent:

[262] 5 juillet - Julien LeNobLe, natif de Blainville ${ }^{168}$, pour Etienne Rassine, habitant de Québec.

[ 263 ] 5 juillet - François LemaiTRE, journalier, de Flers ${ }^{169}, 5$ ans à 60 l. par an, aller et retour payés pour Louis Gaignet, habitant à Quêbec.

[264] 6 juillet - Jean Greves, journalier, mêmes conditions, pour le même, "pour servir en toutes choses". (Teuleron, not. liasse).

\section{4}

à Antoine Grignon, marchand a La Rochelle, faisant pour le sieur Jacques Maheut, de Québec:

[265] 23 mai - Pierre BouanCHEAU, 25 ans, maçon de La Rochelle, pour 3 ans, $60 \mathrm{l}$. par an, avances: 33 1. 5 sols. (Minutes Moreau).

165. Chef-lieu de canton, arrondissement de Mamers (Sarthe).

166. Commune du Canton de Courçon (Charente-Maritime).

167. Commune du canton de Marans, arrondissement de La Rochelle.

168. Soit Blainville (Manche), commune du canton de Saint-Malo-la-Lande; oufsiainville-Crevon, commune du Canton de Buchy, arrondissement de Rouen (Seine-Inférieure).

169. Commune du canton de Domfront (Orne). 
1655

a François Péron, marchand de La Rochelle pour le sieur Bourdon de Québec (Cherbonnier, not. liasse).

[266] 19 juin - Géraud de la Roche, 25 ans, journalier, de Tulle, pour 3 ans à $75 \mathrm{l}$. par an et "si la coutume du païs est que les maîtres fussent obligés de faire repasser leurs serviteurs à leurs despans le temps de son service estant fini ledit sieur Bourdon sera tenu de ce".

[267] François MaRES, 22 ans, journalier, de Tulle.

[ 268 ] 29 juin - Claude Parseval, 35 ans, journalier, de Paris.

[ 269 ] 1er juillet - François TurMEL, 18 ans, d'auprès de Reims. [ 270 ] 2 juillet - Jean Dumayre, 20 ans, journalier, de La Rochelle. [271 ] 3 juillet - Jean PeYronon, 25 ans, tonnelier, de Bordeaux. [ 272 ] 3 juillet - Pierre Lefebvre, 24 ans, filassier, de Bordeaux. [273] 5 juillet - Zacharie FouCHARD, 52 ans, charpentier, de Saint-Pierre d'Oleron ${ }^{170}$.

[ 274 ] 6 juillet - André Bourget 30 ans, maçon, de La Rochelle.

[275] 7 juillet - Jean Dupuy, 45 ans, pâtissier, d'Angers.

[ 276 ] 7 juillet - Pierre Dubry, dit La Verdure, 25 ans, fondeur de Dinant ${ }^{171}$.

[ 277 ] 7 juillet - Jean Groton dit
La Fleur, boulanger, 18 ans, de Châtillon sur Seine ${ }^{172}$.

[278] 7 juillet - Jean BENorst, 24 ans, cordonnier, de Nevers. [279] 8 juillet - Guillaume CouBRET, 22 ans, forgeron, de Bordeaux.

[ 280 ] Jean Midet, 27 ans, journalier, de Troyes.

[281] 30 juillet - Jacques de Presle, 23 ans, chaudronnier, de Rouen, signe.

[ 282 ] François Pasquier, 23 ans, cordier, de La Rochelle.

[283] 2 août - Jean Prieur, 20 ans corroyeur, de Troyes, Signe. [284] 7 août - Jean Houdin, 20 ans, boucher, de Paris.

[ 285] Léonard Bourrelier, 21 ans, cuisinier, de Chartres. Signe.

\section{6}

Engagements par Arnaud Péré pour le sieur Courville, habitant du Canada (Savin, not. Reg.)

[ 286 ] 16 février - Pierre Febvre, laboureur, natif de Beaulieu-surla Roche ${ }^{173}$ (fol. 23).

[287] 17 février - Louis Roger, charpentier de gros œuvre, natif de Vendôme ${ }^{174}$ (fol. 24).

[288 126 mars - Louis Martin, laboureur, demeurant à La Rochelle (fol. $53 \mathrm{v}^{\circ}$ ).

[289] 30 mars - Bertrand PerINEAU, texier, demeurant à La Rochelle (fol. 57).

170. Dans l'île d'Oleron (Charente-Maritime).

171. Vraisemblablement de Dinant, en Belgique.

172. Chef-lieu de canton de la Côte d'Or.

173. Commune du canton de La Mothe-Achard, arrondissement des Sables (Vendée).

174. Loir-et-Cher. 
[290] 3 avril - Jean Tripier, de Sezay Saint-Hilaire, près Vouvant $t^{175}$ en Poitou. Signe. (fol. 58). [ 291-292] Pierre et Thomas Cosson, frères, de Saint-Maurice près La Rochelle, laboureurs (fol. 58 vo).

[293] Bernard Benoist, laboureur, de Saint-Maurice près La Rochelle, (fol. 59).

[294] 7 avril - Jean GLodu, laboureur, de Cognac (fol. 59 $\left.\mathrm{v}^{\circ}\right)$.

[295] 10 avril - François BIBAULT, laboureur de Lafond, près La Rochelle (fol. $60 \mathrm{v}^{\circ}$ ).

[296] 14 avril - Nicolas Picard, laboureur, de Sérigny en Poitou ${ }^{175}$. (Cet acte qui n'est pas signé de l'engagiste, doit sans doute être tenu pour nul). (fol. $63 \mathrm{v}^{\circ}$ ).

à Pierre Mainville, habitant le Canada, s'engage pour 3 ans à 90 1. par an, 30 livres d'avance (P. Moreau, not. Reg. (fol. 77 $\left.\mathrm{v}^{\circ}\right)$.

[ 297 [ 13 mars - André Bouget, 30 ans, maître maçon et tailleur de pierre de La Rochelle. (Cet acte non signé de l'engagiste et paraissant annulé par un: néant final).

à Jacques Pépin, marchand à La Rochelle, s'engagent pour Qué- bec 3 ans (P. Moreau, not. Reg.). [298-299] 23 mars - Jacques Lévesque et son fils Jacques, de La Rochelle, 29 1. par an, un an d'avancé. (fol. $37 \mathrm{v}^{\circ}$ ).

[ 300 ] Louis Blanchard, de Marsillac près Jarnac en Saintong $e^{177}$, 36 livres par an, 431 . d'avance. [301 ] Jean Boutin, 23 ans, de Brouage, 60 l. par an 57 l. 17 sols d'avance.

[ 302 । François Molzeau, 17 ans, des Sables d'Olonne, 36 1. par an, 431 1. 17 sols d'avancées.

[ 303 ] Antoine Pasquier, boulanger, 20 ans, de Poitiers, 60 l. par an, 511.12 s. d'av.

[ 304 ] 24 mars - Léonard MesNIER, laboureur, de La Rochelle, 75 l. par an 45 l. d'av.

[ 305 ] Jean NadaU, laboureur de La Rochelle, 75 l. par an, 37 l. 10 s. d'av.

[ 306 ] Pasquet Nosny, tailleur de pierre, de Salles en Aunis ${ }^{178}, 1001$. 79 l. 14 s. d'av.

[ 307 ] Michel Paroissien, cordonnier, de Saumur, $50 \mathrm{l}$. dont $43 \mathrm{l}$. 17 s. d'av. Signe.

[308] 27 mars - Jean Chauveau dit La Fleur, de Saint-Pierre d'Oleron ${ }^{179}, 84$ l. un an avancé (fol. $95 \mathrm{v}^{\circ}$ ).

[ 309 ] Jacques Journ, dit l'Aiguille, de Chéré en Oleron ${ }^{180}, 84$ l. un an avancé. Signe.

175. Saint-Hilaire, canton de Montaigu, ou Saint-Hilaire, canton de Mortagne-sur-Sèvre (Vendée).

176. Commune du canton de Leigné-sur-Usseau (Vienne).

177. Sans doute Nercillac, commune du canton de Jarnac, Charente.

178. Commune du canton de La Jarrie, arrt. de La Rochelle.

179. En l'île d'Oleron.

180. Cheray, hameau de la commune de Saint-Georges d'Oleron (Ch.-M.). 
[310] Pierre Mrcheau, 38 ans, de Fontenay-le-Comte, 36 l. par an, 38 l. 5 s. d'av.

[311] Jean Crespeau, de SaintVincent-des-Chaimes ${ }^{181}$, faiseur de chars, 60 l. par an, 30 l. d'av. [312] 30 mars - Pierre GenDreau, dit La Poussière, de SaintDenis d'Oleron 84 l. par an, avance: 86 l. 3 s. (fol. $102 \mathrm{v}^{\circ}$ ).

[313] Guillaume Allebert, de Saint-Pierre d'Oleron, 84 l. par an, 87 l. 16 s. d'avance.

[314] Jean Chauvet, 22 ans, de Puilboreau en Aunis ${ }^{182}, 60$ l. par an, 30 l. d'av.

[315] 1er avril - Yves Gaudin, 45 ans, laboureur, de La Rochelle, 75 l. dont $37 \mathrm{l} .10 \mathrm{~s}$. d'av. (fol. 103 vo).

[316] 3 avril - Jean FaURe, maçon de Saint-Julien en Limou$\sin ^{183}, 75$ l. dont $62 \mathrm{l} .13 \mathrm{~s}$. (fol. $\left.105 \mathrm{v}^{\circ}\right)$.

[ 317 ] Jean Delleson, tanneur, de Montauban, 75 l. par an, 52 l. 12 s. d'av.

[318] 5 avril - Pierre Terrifn, 21 ans, de la Jarne en Aunis ${ }^{184}$, 60 l. par an, 40 l. d'av. (fol. 108 vo).

A François Peron "pour Québec et autres endroits du Canada", pour 3 ans à 75 l. par an (pas de mention d'origine). (Cherbonnier, not. Liasse).

[319] 11 avril - François Yvon.
[ 320 ] Charles Achapt, 23 ans, cordonnier, signe.

[ 321 ] Jean Pamier, 28 ans, laboureur.

[ 322 ] Mathieu Dousset, 23 ans, farinier.

[ 323 ] Simon Grenet, 20 ans, laboureur.

[ 324 ] Guillaume Grenet, 22 ans laboureur, cousin germain du précédent.

[ 325 ] Pierre Clément, 29 ans, farinier.

[ 326 ] Jean Millet, 28 ans, laboureur, [ 327 ] Jean Chapron, 21 ans, menuisier.

[ 328 ] Louis Martineau, 27 ans, laboureur.

[329] Jean Forgerat, 30 ans, jardinier.

[330] Pierre Mesnard, 22 ans.

[331] Jean Lafaurest, 25 ans, laboureur.

13321 Jean Nadeau, 23 ans, laboureur. Signe: NADAUlT.

[ 332 ] Denis Renbault, 23 ans, laboureur.

[ 333 ] Antoine Boutelaud, 20 ans, menuisier.

[ 334 ] Jean Rabouin, 18 ans, laboureur.

[ 335 ] Joachim Martin, 20 ans, laboureur.

[ 336 ] André Peuplat, 45 ans, charpentier.

[337 ] Louis Guérineau, 22 ans, journalier.

181. Non identifié.

182. Commune du canton de La Rochelle.

183. Soit Saint-Julien-le-Pélerin (Corrèze) ou Saint-Julien-le-Petit, commune du canton d'Eymoutiers (Haute-Vienne).

184. Commune du canton de la Jarrie, arrondissement de La Rochelle. 
[ 338 ] Guillaume Combret, 23 ans, taillandier.

[ 339 ! Jean Fleurand, 25 ans, laboureur.

[ 340 ] Simon Mineau, 35 ans, journalier.

[ 341 ] Jacques Marchant, 18 ans, laboureur.

[ 342 ] René Vien, 25 ans, laboureur. [ 343 ] Jacques Grassiot, 25 ans, laboureur.

[ 344 ] André Terrien, 25 ans, laboureur.

[ 345 ] Michel Vincent, 20 ans, journalier.

[ 346 ] Nicolas Millet, 29 ans.

[ 347 ] Jacques TruT, 18 ans, tailleur d'habits.

[ 348 ] Jean Laurens, 21 ans, tailleur d'habits.

\section{7}

à Antoine Grignon, Pierre Gaigneur et Jacques Massé, marchands en cette ville, s'engagent pour le Canada, 3 ans (Moreau, not).

[ 349 ] 3 février - Jean Cottereau, 24 ans, de Nieul en Aunis ${ }^{185}, 801$. par an signe.

[ 350 ] Jean Redon, 23 ans, d'Angoulême, 80 l.

[ 351 ] Jacques Fouilleau, 23 ans, de Luçon ${ }^{186}$. Signe 401.

[ 352 ] Elie Riollet, 48 ans, de La Rochelle, 751.

[ 353 ] Jean BÉrisset, 25 ans, de Thaire ${ }^{187}, 901$.
[ 354 ] Michel Touzenu, 28 ans, de La Roche-sur-Yon, 801.

[ 355 ] Laurent Benort, 18 ans, de la Jarne, 751.

[ 356 ] 3 mars - Clément Richard, 23 ans, de Saint-Martin d'Entre$v^{188}, 751$.

[357] Jean Durant, 21 ans de Dieul ${ }^{189}, 751$.

[ 358 ] Jean Coullaud, 18 ans, de Luçon 751.

[ 359 ] Laurent Benoit, 28 ans, de La Jarrie, $75 \mathrm{l}$.

[ 360 ] Pierre Legeron, 18 ans, de Lafond près La Rochelle, 601. [ 361 ] Pierre Biré, 21 ans, de Lafond près La Rochelle, 601.

[362] Jean Rollé, 22 ans, de Nieul en ce gouvernement.

[ 363 ] Grégoire Deblois, 23 ans (pas de lieu d'origine), 701.

[364] Jean BenoIt, 18 ans, de Talmont ${ }^{190}, 601$.

[365 ] François Giron, 18 ans, d'Aytré, 60 livres.

[366 ] Pierre Augereau, 20 ans, de Saint-Maurice, près La Rochelle 70 livres.

[ 367 ] Michel Rocheron, 24 ans, natif de Lagord, près La Rochelle, 801 .

[ 368 ] Nicolas Bouchard, 22 ans, natif de Lagord, près La Rochelle, 801.

[ 369 ] Mathieu Rronteau, 21 ans, de Saint-Eloi près La Rochelle, $80 \mathrm{l}$.

185. Nieul-sur-Mer (Charente-Maritime).

186. Vendée.

187. Commune du canton d'Aigrefeuille (Ch.-M.).

188. Commune du canton de Chef-Boutonne (Deux-Sèvres).

189. Non identifié.

190. Chef-lieu de canton, arrondissement des Sables (Vèndée). 
[ 370 ] Guillaume MÉon, 21 ans, de Saint-Eloi, près La Rochelle, 801.

[ 371 ] Michel Regnaudeau, 21 ans, du Poitou.

[ 372 ] 13 mars - Guillaume BoucQUET, 30 ans, de Chizé ${ }^{191}, 75$ l., dont 38 d'avance.

[ 373 ] 14 mars - Pierre Laurent, 24 ans, de Niort, 60 livres.

[ 374 ] 15 mars - André Pillet, 20 ans, de Tours, 80 livres.

[375] 19 mars - Jean $M_{A Y}, 30$ ans, de Saint-Fort ${ }^{102}, 801$.

[ 376 ] Antoine Serre, 18 ans, de Saint-Amant de Boixe $^{193}, 75 \mathrm{l}$. [377] Mathieu Brunet, 20 ans, d'Olonne ${ }^{194}, 60 \mathrm{l}$.

[378] Michel Le SAN, 18 ans, d'Olonne, $60 \mathrm{l}$.

[ 379 ] Pierre Gourdon, 35 ans, de Fontenay ${ }^{195}, 701$.

[ 380 ] 22 mars - Jean BASSET, 17 ans, de La Rochelle, 751.

[ 381 ] Pierre Guignard, 32 ans, de Saint-Mathurin-sur-la Levée ${ }^{196}$, 751.

[ 382 ] Michel Bouchard, 21 ans, d'Andilly'197 $75 \mathrm{l}$.

[ 383 ] 28 mars - Pierre Allebert, 30 ans, de La Rochelle, 90 l.
[ 384 ] Jean Allebert, 18 ans, de La Rochelle, 901.

[ 385 ] Joachim Reguindeau, 19 ans de La Rochelle, 901.

[ 386 ] 5 avril - Simon MrgnonNEAU, 40 ans, de Montaigu ${ }^{198}, 90 \mathrm{l}$.

[ 387 ] Jean Charpentier, 24 ans, de Nieul ${ }^{199}, 90 \mathrm{l}$.

[ 388 ] Jean Bourasseau, 23 ans, de Saint-Fulgent-en-Poitou, 901.

à Jacques Pichon et à Josué Bestreau, marchands de La Rochelle allant à Québec, (Cherbonnier, not. liasse).

[ 389 ] 15 mars - Jean Charon, 16 ans, de Brouage.

[ 390 ] François Maudet, 20 ans, de Poléon ${ }^{200}$.

[ 391 ] Jérôme Billaudeau, de StMaixent ${ }^{201}$.

à François Péron... pour 3 ans (pas de mention de métier) (Cherbonnier, not. liasse).

[ 392 ] 10 avril - Jean Bourdelais, 23 ans, de Mouchamps en Poitou ${ }^{202}, 60 \mathrm{l}$.

[ 393 ] Etienne Pajon, 32 ans, de Lyon, $75 \mathrm{l}$.

191. Commune du canton de Brioux, Deux-Sèvres, ou hameau de la commune de Vernon (Vienne).

192. Commune du canton de Saint-Denis de Saintonge (Char. Maritime).

193. Chef-lieu de canton de l'arrondissement d'Angoulême (Charente).

194. Près des Sables.

195. Fontenay-le-Comte (Vendée).

196. Commune du canton des Ponts-de-Cé, près d'Angers (Maine-et-Loire).

197. Andilly-les-Marais, commune du canton de Marans (Charente-Maritime).

198. Chef-lieu de commune, arrondissement de la Roche-sur-Yon, Vendée.

199. Nieul-sur-Mer, Charente-Maritime.

200. Village de la commune de Saint-Georges-du-Bois, canton de Surgères (Ch..M.)

201. Chef-lieu de canton des Deux-Sèvres.

202. Commune du canton des Herbiers (Vendée). 
[ 394 ] Antoine Magnan, 35 ans, de La Rochefoucauld ${ }^{203}, 751$.

[ 395 ] Louis Martenot, 21 ans, de Sigournay en Poitou ${ }^{204}$.

[ 396 ] Hilaire Charonnet, 36 ans, de Leray près Fontenay ${ }^{205} 751$. [ 397 ] Jacques Piffray, 22 ans, de La Rochelle, 36 1. puis 42 l. Signe [ 398 ] Mathurin Gouin, 22 ans, de Loudun $^{206}, 451$.

[ 399 ] Laurent Gourn, 20 ans de Loudun, 451.

[400] Marie Pavit, 21 ans, du Château d'Oleron ${ }^{207}, 301$.

[ 401 ] Anne Bouyer, 23 ans, de La Rochelle.

[402] Suzanne Duval, 18 ans, de Soubise ${ }^{208}$.

[ 403 ] Hélène Quartier, 18 ans, de La Rochelle.

[404] Jeanne Garnier, 21 ans, de Clion en Saintonge $e^{209}$.

[405] Marie Drouillard, de 20 ans, de Villemorin ${ }^{210}$, près d'Auray.

[ 406 ] Mathurin Texier, de 18 ans, d'Angoulême, 601.

\section{8}

à Emmanuei Le Borgne fils, pour Emmanuel Le Borgne, père, gouverneur de l'Acadie (Teuleron, not. Reg.).
[ 407 ] 17 janvier - Jean PÉrier, de La Rochelle, comme major, pour 2 ans, à 200 l. par an, $50 \mathrm{l}$. d'av. Aller et retour payés. Signe (fol. 6).

[ 408 ] Jean-Baptiste Frouard, marchand à La Rochelle, natif de Paris, pour deux ans, comme lieutenant de Le Borgne, 200 l. par an, 50 l. d'avance (fol. $6 \mathrm{v}^{\circ}$ ).

[409] René Coullon, marchand de La Rochelle, comme capitaine-enseigne, mêmes conditions. Signe (fol. 7).

à M. Garbuzard, marchand, pour Emmanuel Le Borgne, gouverneur de l'Acadie (Teuleron, not. Reg.):

[ 410 ] 19 février - Jacques LEBouck, marinier, de La Rochelle, 3 ans, à 165 l. par an, 45 l. 5 sous d'avance; passage sur le SaintJoseph, cap. Marot, et retour payê.

'à haut et puissant messire Pierre de Voyer, chevalier, vicomte d'Argenson, gouverneur et lieutenant géneral pour S.M. en la NouvelloFrance" s'engagent à aller 3 ans à Québec "ou autre endroit du Canada, au service, fidélité et obéissance dudit seigneur ou de ceux de ses officiers qu'il leur

203. Chef-lieu de canton, de la Charente.

204. Commune du canton de Chantonnay (Vendée).

205. Non identifié.

206. (Vienne).

207. Chef-lieu, canton de la Charente-Maritime, dans l'sle d'Oleron.

208. Commune du canton de Saint-Agnant, Charente-Maritime.

209. Commune du canton de Saint-Genis, de Saintonge (Charente-Maritime).

210. Commune du canton d'Aulnay (id.). 
ordonnera au fait de la milice or à autre emploi honnête et civil..." au prix de 75 l. par an (Cherbonnier, not.

[411] 12-20 avril - Pierre GIL BERT, dit La Marche, habitant de cette ville, 35 ans, 30 l. d'avance. Signe.

[ 412 ] Jean LÉgier dit La Tour, de Coutras en Gascogne, 20 ans, 301. d'av. Signe.

[413] François Girard, sieur du Foy, natif de Laneuville en Beauvaisis $^{211}, 28$ ans, 30 l. d'av. Signe. [ 414 ] François Priqué, 17 ans, de Paris, 30 l. d'av.

à Pierre Denis, écuyer, à La Rochelle, pour Jacques de la Poterie à Québec, passage sur le PierreGuillaume. (Teuleron, not. Reg.).

[415] 16 avril - Jeanne Perrin, femme de Pierre Dutaud, portefaix à La Rochelle et avec son autorisation, pour 5 ans, à $50 \mathrm{l}$. par an, comme servante. Aller et retour payés.

[416] Madeleine Dutaud, leur fille âgée de 9 ans part avec elle.

[417] Charles Dutaud, leur fils, âgé de 16 ans, pour 3 ans, à 301 . par an, aller et retour payés.

[418] 17 avril - Marie Dutaud, 19 ans, leur fille, pour 3 ans à 40 l. par an, aller et retour payés.

Engagements par Pierre Gaigneur, pour le Canada (Moreau, not.)
[419] 30 avril-2 mai - Thomas Marchant, farinier, de Marsay en Aunis ${ }^{212}$.

[ 420 [ Pierre Frichet, de La Rochelle.

[421] François Quillet, farinier, de La Rochelle.

[422] André Coudret, de SaintFort de Conat en Saintonge ${ }^{213}$. [ 423-424 ] Jacques Chappelin, père et Jacques, son fils, tourneurs et menuisiers de La Rochelle.

[ 425 ] Jean Pinsart, laboureur, de PÉRIGNY ${ }^{214}$ (testament au fol. suivant.

[426] Mathurin Bellouard, de Romagné ${ }^{215}$.

A Jacques Vivien, marchand à LaRochelle, pour Jean Sauvaget, habitant à Québec (Moreau, not.)

[ 427 ] 30 avril - André Bonneau, de Vouhé en Aunis ${ }^{216}$.

à Pierre Sadot et à Henri Desmoulins, écuyer, demeurant ordinairement à Paris, pour Québec, 3 ans, aller et retour (Teuleron, not. Reg.)

[428] 10 mai - Charles NarP, fils de Simon et de Jacq iuette Baron, qui se porte garant du départ de son fils. Signe.

à Arnaud Pérépour Pierre Boucher $\mathbf{S}^{r}$ de Grois-Bois, demeurant aux Trois Rivières (Savin, not. Reg.) (Oise)

211. Laneuville, hameau de la Commune de Moliens, canton de Formerie

212. Commune du canton de Surgères (Charente-Maritime).

213. Saint-Fors de Cognac (Charente), commune du canton de Segonzac.

214. Commune du canton de La Rochelle (Ch.-Maritime).

215. Hameau du canton de Saint-Xandre (Charente-Maritime).

216. Commune du canton de Surgères (Ch.-Maritime). 
[429-430] 11 mai - Etienne GEL LINEAU, sargier et charpentier de gros ouvre et Jean, son fils, de Saint-Vivien de Pons ${ }^{217}$.

Engagements par François Péron, à Québec "pour lui ou autres", sur le Taureau; aux gages de $75 \mathrm{l}$.

par an, 35 1. d'avancé. (Cherbonnier, not. Liasse).

[ 431] 14-15 mai - Nicolas ChAVIGNEAU, 18 ans, de Lumbertière en Aunis ${ }^{218}$.

[ 432 ] Jean Nepardeau, 32 ans, de Saint-Amand de Bongneux, châtellenie de La Rochefoucauld ${ }^{219}$

[ 433 ] Isaac Lalleman, 16 ans, du Breuil de Lisle, près de SaintJean d'Angély ${ }^{220}$.

[ 434 ] Nicolas MASSard, du grand Hivresay, paroisse de Vouille ${ }^{221}$ près Poitiers.

[ 435 ] Michel Ballanger, 30 ans, de Varaize près Saint-Jean d'Augély 222 .

[ 436 ] Francois Rou, 25 ans, de Haut-le-Moutier, près Loches ${ }^{223}$.

[ 437 ] Pierre Mourrier, 22 ans, de Chenove (?) près Ruffece ${ }^{224}$.

[ 438 ] Nicolas Giard, 23 ans, de la
Coussardière, paroisse de Melloran près Chef-Boutonne $e^{225}$.

[439] Yvon RAssavonin, 20 ans, de Plouarde, près Brest ${ }^{26}$.

[ 440 ] Mathurin GUICHARD, 40 ans, de Fontenay-lo-Comte.

[ 441 ] Pierre Millet, 40 ans, de Fontenay-le-Comte.

[ 442 ] Pierre Motre, 45 ans, de la Rochelle.

[443] Jean Allaire, 23 ans, de Saint-Philbert en Poitou ${ }^{227}$.

[ 444 ] Louis Broussard, 28 ans, de Beaulieu en la Châtellenie de Sensat en Saintonge $e^{228}$.

[ 445 ] Charles Allaire, 18 ans, de Saint-Philbert.

[ 446 ] Mathurine Lacroze, âgée de 22 ans, de Niort, aux gages de 37 1. 10 sols.

1659

à Guillaume Vignal, prêtre logé au logis du Dauphin, à La Rochelle, paroisse Saint-Nicolas, s'engagent de passer à Québec sur le SaintAndré, pour 3 ans (Cherbonnier, not).

[ 447 ] 11 mai - Respin Cochelier, compagnon menuisier, de Cler-

217. Paroisse de Pons (Charente-Maritime).

218. Non identifié.

219. Saint-Amant de Bonnieure, commune du canton de Mansle (Charente).

220. Village de la Commune de Saint-Pierre de l'Isle (Char.-Maritime).

221. Canton de Vouille (Vienne).

222. Commune du canton de Saint-Jean d'Angély (Char.--Maritime).

223. Non identifié.

224. La lecture est douteuse.

225. Commune du canton de Sauzé (Deux Sèvres).

226. Non identifié.

227. Sans doute Saint-Philbert de Bouaisne (Vendée) commune du canton de La Rocheservière.

228. Soit Beaulieu, commune de Dignac, soit Beaulieu, commune d'Etagnot. l'une et l'autre en Charente. 
mont en Beauvaisis ${ }^{229}, 29$ ans, "travaillant présentement de son travail en la boutique de Guillaume Vergalier, maître menuisier et sculpteur de cette ville, qui servira les sœurs Ursulines de son métier ou d'un autre emploi honnête", à 110 l. par an, avec tous les outils qui lui seront fournis. $40 \mathrm{l}$. d'avance.

[448] 19 mai - Noël Legall, compagnon menuisier, du Conquet, pays de Bretagne ${ }^{230}, 22$ ans pour Québec ou Montréal. Il servira Vignal ou un autre, en son métier ou en autre emploi à $80 \mathrm{l}$. par an, 40 l. avancées.

à Jeanne Manse, pour l'Hôtel-Dieu de Montréal, s'engagent:

[ 449 ] 5 mai - Pierre Perusseau, laboureur, de Saint-Jean d'Augély.

[ 450 ] Grégoire Simon, de la Mauguignière, de Benay, près Civray $^{231}$.

[451] 8 juin - Jean Celier, do Riom en Auvergne ${ }^{232}$.

[ 452 ] René Cuillerier, de Clermont près la Flèche en Anjou.

[ 453 ] 12 juin - Jacques Laval, de Paris.

[ 454 ] Pierre Moreau, de La Rochelle.

[ 455 ] Jacques Boumrn "de la paroisse delle"233.

[ 456 ] Noël Davignon, maître maçon de La Rochelle.
[457] 18 juin - Marie Poléon, signe.

à la même obligations pour passage:

[458-459] 5 juin - Olivier CHERbonneau et Marie Garnier, sa femme.

[ 460-461 ] Pierre Goyer et Louise GarNier.

[462-463] Simon Cardinault et Michèle Garnier.

[ 464-465] Jean Leroy et Frangoise Bovet.

[ 466-467] Mathurin Thibaudeau et Catherine Crat (?)

[ 468-469] Jean Racault et Renée Bovet.

[ 470-471 ] Pierre Guiberge et Mathurine Desbordes.

[ 472-473 ] Elie Boson et Suzanne Cougnon.

Engagements ou obligations pour passage à Jørôme Le Royer pour l'abbé de Caylus de Montréal (Demontreau, not. Reg.).

[ 474-475] 8 juin - Claude FezeREt et Suzanne Guillebault, son épouse.

[ 476 ] André Bourget, mą̧on et tailleur de pierre.

[ 477] Jean Condrat, maçon et tailleur de pierre

[478] Gilles de Vime, maçon et tailleur de pierre.

[ 479 ] Jacques Mestivier, maçon et tailleur de pierre.

[ 480 ] Jacques Marsetteau, magon et tailleur de pierre.

229. Oise.

230. Commune du canton de Saint-Renan, Finistère.

231. Commune du canton de Champagne-Mouton (Charente).

232. Puy-de-Dôme.

233. Elle, l'Ile d'Elle (Vendée), commune du canton de Chaillé-les-Marais. 
[ 481 ] Mathurin Marsetteau, macon et tailleur de pierre.

[ 482 ] Etienne Truteau, charpentier, de La Rochelle.

[ 483-484 ] Henri Bailly, maître maçon et entrepreneur de bâtiments et Marie Fonteneau, sa femme.

[ 485 ] René Moreau, de Clermont en Auvergne.

[ 486 ] Etienne Hardouin, de Clermont en Auvergne.

[487] Jacques Le Pretre, du Loreau-Beconnais, près d'Angers ${ }^{234}$.

[ 488 ] Julien Averty, de la Flèche

[ 489 ] Pierre Neveu, de Béré près d'Angers ${ }^{235}$.

au même pour M. Souard, prêtre de Montréal (même notaire).

[490] 8 juin - Jean Renou, de Mézéré au Maine $^{236}$.

[491] Jean Martineau, de SaintGermain du $\mathrm{Val}^{237}$.

[ 492 ]Antoine Courtimanche, de Saint-Pierre de Chavillée ${ }^{238}$.

à Claude Rabussel, sieur de SaintAndré, pour Montréal (Demontreau, not. Reg.).

[493] 8 juin - Michel Bonnier, maçon, de La Flèche.
[494] Jean Leblanc, maçon, de La Flèche.

à Jacques Mousnier, pour Montréal ou Québec (même not.).

[495] 26 juin - Jean Augrin, du bourg de Counnes (? $)^{239}$ au Bas-Maine.

[496] 30 juin - Jacques Millet, défricheur et faiseur de treilles, du village de Bourgain, paroisse de Bourneau en Bas Poitou ${ }^{240}$.

Engagements pour Québec à Francois Pêron, pour 3 ans à $75 \mathrm{l}$. par an (Cherbonnier, not. Liasse).

[ 497 ] 27 juin - Mathurin REGUE$\mathrm{NY}, 25$ ans, tonnelier et laboureur, de Ré.

[ 498 ] Jacques Grimaux, 23 ans, laboureur, de Champigné le Sec à une lieue de Mirebeau,en Anjou ${ }^{241}$.

[ 499 ] Elie Charrier, 37 ans, tanneur des Herbiers, 801 . par an. ${ }^{242}$.

[ 500 ] Jean Mathiev, 23 ans, do Tapy en Angoumois ${ }^{243}$.

[501 ] Jean Brothier, 18 ans, d'Aytré.

à Emmanuel Le Borgne, ehevalier, de La Rochelle, faisant pour lo sieur du Coudray, lieutenant pour Loire).

234. Le Louroux-Béconnais, canton de l'arrondissement d'Angers, (Maino-et-

235. Peut-être le hameau de Bère, commune de Chateaubriant (Loire-Inf.).

236. Mézeray, commune du canton de Malicorne (Sarthe).

237. Commune du canton de La Flèche (Sarthe).

238. Saint-Pierre de Chevillé, commune du canton de Château-du-Loir (Sarthe).

239. Peut-être Conneré, commune du canton de Montfort (Sarthe).

240. Le Bourneau, commune du canton de l'Hermenault (Vendée).

241. Mirebeau, chef-lieu de canton de la Vienne.

242. Chef-lieu de canton de la Vendée. (Ch.).

243. Hameau de la commune de Montignac, canton de Saint-Amant de Boixe 
le roi de la Nouvelle-France (Teuleron, not. Reg. 1659-1661). [502] 25 février -- Pierre MerCIER, chirurgien de Neufchâteau près Montfort l'Amaury, en Beau$\mathrm{ce}^{244}$, pour un an, à $80 \mathrm{l}$. par an. aller et retour payés, "tout lui étant fourni", il pourra travailler pour lui en temps libre. Signe.

à Antoine Grignon, marchand à La Rochelle, faisant pour Eustache Lambert, de Québec, pour 3 ans à 80 l. par an (P. Moreau, not. Reg. $\mathrm{Nv}^{\circ}$ ).

[503] 20 avril - André RoberDON, 25 ans, meunier, de Nantes.

à Michel Pelletier, sieur de la Prade, habitant des Trois-Rivières s'engage pour 3 ans, comme charpentiers "ou autre choses". (P. Moreau, not. Reg. fol. $104 \mathrm{v}^{\circ}$ ). [504] 6 mai - Abraham CAILLAUD, maître charpentier de gros œuvre de La Rochelle, à 100 l. par an, 70 l. d'avance. Caillaud emmenera ses outils.

[ 505 ] Daniel Chargneau, garçon charpentier de gros œuvres de La Rochelle à 65 l. par an, $30 \mathrm{l}$. d'av.

à Médard Chouart, sieur des Groseillères, général de la flotte des Autaois (Ottawa) demeurant aux Trois-Rivières, pour 3 ans (Moreau, not. Reg.).

[ 506 ] 24 mai - Jean Crespeau, 24 ans, laboureur, de Laleu pròs La
Rochelle, à 78 l. par an. 391 . d'avance (fol. $118 \mathrm{v}^{\circ}$ ).

[ 507 ] 27 mai - René VALLet, 24 ans, arquebusier, de Saumur, a 75 l. par an, "pour y exercer sa profession ou autres choses commandées"... "le dit Chouart a accordé à Christophe Garbault, soldat de la garnison des TroisRivières... de le recevoir à boire et à manger en sa maison avec ledit Vallet, son cousin, en apportant en ladite maison les provisions qui lui seront données dans ladite garnison pour sa nourriture". Signent Chouart et Gerbault. (fol. $122 \mathrm{v}^{\circ}$ ).

[ 508 ] 17 juin - Antoine D'Aulnay, 23 ans, laboureur, de Luçon (fol. 135).

[509] Louis Gaborit, 22 ans, de Saint-Martin de la Coudre près Paransay en Saintonge ${ }^{245}$. Signe. [510] Etienne Vergnonneau, 21 ans, de Bonnevault en Poitou ${ }^{246}$, 72 livres par an.

à Jean Bourdon, sieur de SaintFrançois, habitant de Québec, pour 3 ans à $72 \mathrm{l}$. par an, mais ces actes ne sont pas signés (Teuleron, not. Reg.).

[ 511 ] 15 juin - François Drouet, 24 ans, de Corbenais, près Paris ${ }^{247}$.

[512] Pierre Drouet, 24 ans.

[513] Jacques Gordin, 30 ans, de Saint-Pierre de la Selle près Poitiers.

[514] Jean Goidin, 30 ans, de

244. Monfort-d'Amaury, chef-lieu de canton de la Seine \& Oise.

245. Saint-Martin de la Coudre, commune du canton de Loulay (Ch. Maritime).

246. Non identifié.

247. Sans doute Corbény (Aisne), commune du canton de Craonne. 
Saint-Pierre de la Selle près Poitiers. ${ }^{248}$

[ 515 ] Jean Fabertas.

à Médard Chouart, pour François de la Chapelle, habitant des TroisRivières (P. Moreau, not. Reg.). [ 516 ] 17 juin - Pierre Romieux, dit Romellus, 23 ans, chirurgien, de Béziers en Languedoc ${ }^{249}$, pour 3 ans, comme chirurgien, à 2001 . par an, pourra travailler pour son usage. La Chapelle fournira les médicaments. Signe (fol. $135 \mathrm{v}^{\circ}$ ).

\section{2}

au $\mathrm{s}^{\mathrm{r}}$ Savager, marchand habitant du Canada pour le $\mathrm{s}^{\mathbf{r}}$ Boucher marchand au Canada (Teuleron, not. Reg. 1661-1663).

[ 517 ] 1er mai - Edmond Henry sr du Chasterie, de Troyes en Champagne, pour 3 ans; recevra 20 sols par jour jusqu'au départ. Pas d'indication de gages, ni de signatures. A la place des gages un blanc a été laissé.

[ 518 ] Pierre Poullard, de Mortagne au Perche, id.

[519] 2 mai - Abel Sagot, de Champ-Frémont dans le Maine ${ }^{250}$. Les frais de séjour à La Rochelle lui sont payés à la journée depuis le 6 mars précédent. Ils le seront jusqu'à l'embarquement.

à Simon Denis, écuyer, sieur de la Trinité, demeurant à Québec au
Canada, pour 3 ans, comme meunier (P. Moreau not. Reg.).

[ 520 ] 9 mai - Mathurin GRAIN, 32 ans, meunier, de Saint-Laurent de la Salle, près Sainte-Hermine en Poitou ${ }^{251}$, 100 l. par an (fol. 117 $\mathrm{v}^{0}$ ).

à Pierre Gaigneur, marchand à La Rochelle, faisant pour les PP. Jésuites de Québec, pour 3 ans et pour exercer son métier de meunier (P. Moreau not. Reg. fol. 116 $\left.\mathrm{v}^{\circ}\right)$.

[521] 9 mai - René Blanchet, 23 ans, de Fontaines ${ }^{252}$ près Fontenay-le-Comte, meunier, 1001. par an, retour payé.

à Pierre Boucher, sieur de GrosBois en la Nouvelle-France, demeurant aux Trois-Rivières, s'engage pour 3 ans pour l'apprentissage de charron et de charpentier (P. Moreau, not. Reg. fol. 143 vo). [ 522 ] 17 juin - Pierre Dancosse, 18 ans, de La Rochelle.

\section{3}

Liste de l'équipage du Thoros, de la ville de La Rochelle (6 canons, 5 mousquets) pour le Canada Arch. Char. Marit, B 5664, pièce 138 bis).

[ 523 ] 5 mai 1663 - Pierre LaMBERT, de Tours.

[524] Guillaume Bernard, d'Angers.

248. Paroisse de Poitiers (Vienne).

249. Chef-lieu d'arrondissement de l'Hérault.

250. Commune du canton de Pré-au-Mail (Mayenne).

251. Sainte-Hermine, chef-lieu de canton de la Vendée.

252. Commune du canton de Fontenay-le-Comte (Vendée). 
[ 525 ] Jean (?) Thomas, de Poitiers. [526] Daniel Olier, serrurier, de Saumur.

[ 527 ] Charles Bouier, laboureur, de Parthenay.

[ 528 ] Mathurin Poirier, de Florent de la Salle, en Poitou ${ }^{253}$. [ 529 ] Pierre Gosrion, d'Aigre ${ }^{254}$. [ 530 ] Pierre Loran, de Tours.

1664

à Emmanuel Le Borgne, le jeune, sr du Coudray, demeurant à La Rochelle, s'engage à partir sur la Paix, de $200^{\text {tx }}$, $\mathrm{c}^{\mathrm{ne}}$ Elie Siberon, pour le Canada, pour 3 ans, aller et retour, 80 livres par an 30 livres d'avance (Teuleron, not. 16631666).

[531] 18 fóvrier Jean Benort, maître armurier, de La Rochelle.

Liste des hommes de l'esquipage du navire Noir, de Hollande, $\mathrm{e}^{\mathrm{ne}}$ Pierre Filly, de Dieppe (Arch. Char. Mar. Amirautê, B 5665, pièce 10$)^{255}$.
[ 532 ] Jean Gres 256 , de Nerpon, au pays nantais ${ }^{257}$.

[533] Antoine Pajau, de Ruffec, en Poitou.

[ 534 ] Joachim Brunet, de La Rochelle.

[ 535 ] Mathelin Cardin, de SainteSoulline en Poitou 258 .

[ 536 ] Pierre Blet, de Chef-Boutonne $e^{259}$.

[ 537 ] Jacques Duboys, de Nieul en Poitou ${ }^{260}$.

[ 538 ] Marc Butain, de Paris.

[ 539 ] Simon Gilleret, de Blois.

[540] Mathurin MoreaU, do Champdeniers, en Poitou ${ }^{261}$.

[ 541 ] Jacques Gerny, de l'Aiguil$\operatorname{lon}^{262}$.

[ 542 ] Pierre Audigé.

[543] François Marchand.

[ 544 ] Pierre Houdan, dit La Taille, de Lisieux ${ }^{263}$.

[ 545 ] François Hilleret, de Marans.

[546] René Peltier, de Marans. [ 547 ] Simon Derne, de La Rochelle. [ 548 ] Nicolas Boué, du Poiro-doVelluire ${ }^{264}$.

253. Saint-Florent de la Salle, sans doute, non identifié.

254. Chef-lieu de canton de la Charente.

255. Cette liste est sans date. C'est le P. Godbout qui a découvert la destination du navire. Il a aussi identifié la plupart de ces engagés, dans un article à paraître dans les Mémoires de la Societé généalogique canadienne-française.

256. Ou GRIS.

257. Non identifié.

258. Commune du canton de Brossac, Charente.

259. Chef-lieu de canton des Deux-Sèvres.

260. Il est difficile de savoir s'il s'agit de Nieul-l'Espoir, dans la Vienne, de Nieulle-Dolent, ou de Nieul-sur-l'Autise, en Vendée.

261. Chef-lieu de canton des Deux-Sèvres.

262. Ou Aiguillon-sur-Mer, commune du canton de Luçon (Vendée) ou Aiguillon sur-Vie ou du canton de Saint-Gilles-sur-Vie (Vendée).

263. Calvados.

264. Commune du canton de Fontenay-le-Comte (Vendée). 
[ 549 ] Andre Giloche, du Guఠ-deVelluire ${ }^{255}$.

[550] Jean Fretré, du GuédeVelluire.

[ 551 ] Pierre Cailleau, du GuédeVelluire.

[ 552 ] Pierre Fourníe, d'Aigre, en Poitou.

[ 553 ] Jean Fourment, de Monem$\mathrm{dy}^{266}$ en Picardie.

[ 554 ] Louis Delahaye, de Loudun.

[555] Antoine Auton, d'Angoumois.

[ 556 ] Jean Pollican, de Loumaria, en Bretagne ${ }^{267}$.

[ 557 ] Pierre Riveau, de Ruelle, en Angoumois ${ }^{288}$.

[ 558 ] Nicolas Fournier de Marans.

[ 559 ] Antoine TapIN, du Poiré-surVelluire.

[ 560 ] André Gautron, de La Rochelle.

[561] Mathelin Corniveau, de Fontenay.

[ 562 ] Jean BAUdeT, de Blanchet en Poitou ${ }^{269}$.

[ 563 ] Jean Bertran, d'Aubenas ${ }^{270}$. [564] Jean Plaignol, d'Aubenas.

[ 565 ] François BoA, de Villegagnon.

[ 566 ] Jean Roy, de Lagny ${ }^{271}$.

[ 567 ] Jean Robert, de Lagny.

[ 568 ] Pierre Groulet.

[ 569 ] Toussaint BAUDRY, de Lagny.

[570] Jeanne Benart, de Paris.

[ 571 ] Jean Barluie, de l'île de Ré.
[ 572 ] Jacques BAudouin.

[ 573 ] Nicolas Geoffroy, de l'ŝle de Ré.

[ 574 ] Pierre Parot, de l'île de Re.

[ 575 ] Rene Brisson, de La Rochelle

[ 576 ] Frangois BASTARd, de Jaun$n \hat{\sigma}^{272}$ en Poitou.

[577 ] Jaeques Maignard, de La Rochelle.

[578 ] Jean Bouesmé, de Poitiers.

[ 579 ] René Jouchon, de Poitiers.

[ 580 ] Mathias Compagnat, de La Rochelle.

[ 581 ] Pierre Compagnat, de La Rochelle.

[ 582 ] Charles Combault, de SaintJean d'Angély.

à Pierre Gaigneur, marchand à La Rochelle, pour le Canada, passage sur l'Aigle Blanc, de Flessingue, 200 tx, ene André Chaviteau (Teuleron, not. Reg. 1663-1664). [ 583 ] 28 avril - Jean CADET, chirurgien, de Parthenay, pour servir pendant un an le sieur de Boispeau, commandant de l'habitation de Quanseau en Nouvelle France.

\section{5}

au même pour les PP. Jésuites de Québec. (Teuleron, not. Reg. fol. $79)$.

[ 584 ] 2 avril - Jacques Roche, de

265. Commune du canton de Chaillé-les-Marais (Vendée).

266. Serait-ce Montmédy (Meuse)?

267. Commune du canton du Palais (Morbihan).

268. Commune du canton d'Angoulême (Charente).

269. Non identifié.

270. Ou Aubenay?

271. Chef-lieu de canton de la Seine-et-Marne.

272. Jaulnay, commune du canton de Saint-Georges (Vienne) ou Jaunay, hameau de la Commune d'Azay-le-Brule (Deux-Sèvres). 
la Charrière ${ }^{273}, 3$ ans, 75 l. par an, 37 d'avance.

au même, pour le sieur Lambert, marchand à Québec (id. fol. 80).

[585] 2 avril - Pierre Damien, de La Flotte-en-Ré, 3 ans, à 75 l. par an, 30 l. d'avance.

au même pour Jean Grignon, marchand à Québec (id. fol. 81).

[586 ] 3 avril - Jacques BoIsson, natif de Ré, 3 ans, 80 l. et 45 l. d'av.

au même, pour Louis Latour, bourgeois de Québec (id. fol. 95).

[ 587 ] 18 avril - Pierre Martin, de Villeneuve-la-Comtesse ${ }^{274}$ en Poitou, 3 ans, 90 l. par an 30 l. d'av.

[588] Guillaume CANAT, né à Sigournais en ce gouvernement, id.

au même, s'engagent à partir pour Québec sur le Cat de Hollande cne Charles Babin (Teuleron, not.) pour 3 ans, tous reçoivent $30 \mathrm{l}$. d'avance.

[ 589 ] 23 mars - Jean Blanchard, de Surgères ${ }^{275}, 601$.

[ 590 ] Bastien Roy, de Loudun, 70 l. [ 591 ] Mathieu LaUrendin, de Loudun, 601 .

[ 592 ] René Binet, de Loudun, 601.
[ 593 ] Etienne Renaud, de Villegagnon, $90 \mathrm{l}$.

[594] 26 mars - Louis PaLARdy, de Saint-Mars des Préss ${ }^{276}, 751$.

[ 595 ] Simon Trillaud, du bourg d'Ambars ${ }^{277} 60 \mathrm{l}$.

[ 596 ] Pierre Pasquier, de SainteSoulle $^{278} 751$.

[ 597 ] Jacques Boussot, de la Rochelle. $75 \mathrm{l}$.

[ 598 ] 27 mars - Etienne RichaRDEAU, de Chambon ${ }^{279}, 75 \mathrm{l}$.

[ 599 ] René Giraudet, de présent à La Rochelle 801.

[600] Pierre Francois, faiseur de sabots 751 .

[ 601 ] Jean Roy, d'Aytré, 901.

[ 602 ] Jacques Restié, de la Choinière $e^{280}, 75 \mathrm{l}$.

[603] Jean Guillet, de la Flotte en Ré, 80 l.

[ 604 ] Julien Allard, de la Flotte en Ré, $80 \mathrm{l}$.

[605] Simon Gendron, de SaintNicolas ${ }^{281}, 801$.

[ 606 ] André Fouquet, d'Aytré, 801.

[607 ] Jean Caillon, d'Aytré, 80 l. [ 608 ] Pierre Caillon, d'Aytré, 801. [609] 28 mars - Simon Lassalle, de la Rochelle, $75 \mathrm{l}$.

[610 ] Pierre Alleret, de Marans, $75 \mathrm{l}$.

273. Commune du canton de Beauvoir (Deux-Sèvres).

274. Commune canton de Loulay (Charente-Maritime).

275. Chef-lieu de canton de la Charente-Maritime.

276. Commune du canton de Chantonnay (Vendée).

277. Ambax? (Haute-Garonne) commune du canton de l'Isle-en Dodon.

278. Commune du canton de La Jarrie, (Charente-Maritime).

279. Commune du canton d'Aigrefeuille (Charente-Maritime).

280. Commune du canton de Beauvoir (Deux-Sèvres).

281. Sans doute Saint-Nicolas de la Rochelle. 
[ 611 ] Jacques Bourdin, de SaintJean d'Angély $75 \mathrm{l}$.

[612] 30 mars - Jacques Borsson, de La Flotte, 801.

[ 613 ] Jean Court, de La Rochelle, 751.

[ 614 ] 31 mars - Denis Genty, menuisier, de présent à La Rochelle, 901.

[615 ] Jean Grenet, d'Aytré, 751.

[616 ] Jacques Rousseau, de Loix en l'île de Ré, 751.

[617] Mathurin Villeneuve, de Sainte-Marie ${ }^{282}$, à présent en cette ville, 751.

[618] François Thibaud, de la Flotte, 751.

[619] Jean Guillet, de la Flotte, avances de 501.801.

[ 620 ] Jean Bessonnet, de la Flotte, 751.

[ 621 ] Pierre Mercereau, de SaintMartin de Ré, de présent à la Rochelle, 751.

[622 ] Gilles Gadiou, de la Flotte.

[ 623 ] Pierre Nepveu, d'Ars en Ré.

[ 624 ] Pierre Messager, de la Flotte.

[ 625 ] Gilles Gautrais, de la Flotte.

[626] 1er avril - Jacques CHEvalier, de la Flotte.

[627 ] Gilles du RAY, de SainteMarie de Ré, 75 l. av. 35 l.

[628] Guillaume Bertrand, de Sainte-Marie de Ré, 75 l. av. 35 l.

[629] Jacques Crespaud, de Lafond près La Rochelle, 601.
[630 ] Pierre Mercier, de SaintDenis, en Poitou ${ }^{283}$.

[ 631 ] Antoine MÉrit, de Mauzéers. [ 632 ] Louis Bonnet, de Noëze ${ }^{285}$.

[633 ] Nicolas Pion, né à Thurageau $^{286}$.

[634 ] Pierre Elbert, de SaintMartin de Ré, $75 \mathrm{l}$.

[635] 2 avril - Pierre CaIllonNEAU, de Sainte-Marie de Ré, 751.

[636 ] Pierre Luinas, de SainteSévère ${ }^{287}, 751$.

[ 637 ] Marc Texier, de La Rochelle, 601.

[638] Martin Chevalier, de la Flotte, 751 . avance $45 \mathrm{l}$.

[639] 3 avril - René Orzeau, maçon de la Flotte, 751.

[640] Jacques Courterl, de La Rochelle, 751.

[641] Nicolas Villeneau, de la Flotte, 751.

[642] René Rezeau, de Ré, 75 l.

[ 643 ] Alexis Bries, de la Flotte, 75 livres.

[ 644 ] Nicolas Bustort, de Ré, 75 l.

[ 645 ] Simon La Salle, de La Rochelle, 751.

[646 ] Pierre Genaudeau, de Re, 751.

[ 647 ] Pierre Caillonneau, de Rivedoux ${ }^{288}, 751$.

[648] Mathurin Motel, de Saumur,70 1.

282. Sans doute Sainte-Marie, commune du canton de Chalais, en Charente.

283. Sans doute Saint-Denis-la-Chevasse, commune du canton de Poiré-surVic (Vendée).

284. Chef-lieu de canton des Deux-Sèvres.

285. Commune du canton de Saint-Aignant (Ch. Maritime).

286. Commune du canton de Mirebeau (Vienne).

287. Commune du canton de Jarnac (Charente).

288. Dans l'île de Ré (Ch. Maritime). 
[649] Laurent Dron, de SaintXandre ${ }^{289}, 75 \mathrm{l}$.

[650 ] Jean Bertain, de Sisse $e^{200}$, 751.

[651 ] Jacques Damien, de La Flotte, 761.

[ 652 ] 4 avril - François Fortage, de La Flotte, 751.

[653 ] Jacques Rifort, de SaintJean d'Angély, $70 \mathrm{l}$.

[654] 18 avril - Antoine DelaFosse, de présent à La Rochelle, 751.

[655] Nicolas RagnaU, de présent à La Rochelle, $75 \mathrm{l}$.

aucun d'eux ne signe.

Engagement:par Daumont de SaintLusson, prêt d'aller habiter à Québec (Lefebvre, not. Reg. 16641665, fol. 54).

[656] 20 juin 1665 - Maurice Olivier, laboureur, de Rioux en Saintonge ${ }^{291}$ âgé de 27 ans, pour 3 ans, à 45 l. pour les 3 ans.

1666

à Pierre Gaigneur, marchand de La Rochelle, faisant pour le sieur Lambert, habitant, à Québec (Teuleron, not. Reg. fol. $71 \mathrm{v}^{\circ}$ ). [657 ] 14 avril - Urbain Hulin, 45 ans, du Puy-Notre-Dame en $\mathrm{Anjou}^{292}$, pour 3 ans, à 901 . par an, nourri jusqu'à l'embarquement "pour servir en toutes choses, sauf à labourer la terre". Signe. au même, au nom de MM. de la Compagnie des Indes Occidendales au Canada (Teuleron, not. Liasse).

[658 ] 1er mai - Jacques Tessier, laboureur, de Nantes, 75 l. par an, pour 3 ans.

\section{7}

à Pierre Gaigneur, pour la Compagnie des Indes Occidentales, a Québec, sur la Constance, de 300 tx cap. André Pasquinet (Teuleron, not. Liasse).

[ 659 ] 4 avril - Louis Ballon, de la Rochelle, 60 l. par an, $30 \mathrm{l}$. d'avance, nourri jusqu'à l'embarquement. Signe.

[660] 17 avril - Martin Florat, jardinier de Paris, à $70 \mathrm{l} .30 \mathrm{l}$. d'avance. Signe.

[661 ] 17 mai Michel Coquelin, tailleur d'habits, du Mans, 21 ans, 75 l. Signe.

i 662 ] 19 juin - Jean GuY, laboureur, de Saint-Jean d'Angély, 70 l. par an.

[ 663 ] 22 juin - René Fourault, journalier, de La Flèche, 22 ans, 60 l. par an. Signe.

[ 664 ] 30 juin - Martin Mercier, journalier, des Sables, 19 ans, 60 l. par an. Signe.

à Arnaud Péré, marchand à La Rochelle, pour les PP. Jésuites de Québec (Teuleron, not. Liasse).

289. Commune du canton de La Rochelle (Ch. Maritime).

290. Sissy (Aisne) Sisas (Haute-Vienne ou plutôt Cissé (Vienne).

291. Commune du canton de Gémozac (Char. Maritime).

292. Commune du canton de Montreuil-Belloy (Maine-et-Loire). 
[665] 12 avril - Pierre Arnaud, laboureur à bœufs, de SaintPierre d'Excideuil, près Civray en Poitou ${ }^{293}, 45$ ans, pour 3 ans, à $75 \mathrm{l}$. par an, $40 \mathrm{l}$. d'av., nourri jusqu'à l'embarquement.

[666 ] Pierre Francors, demeurant à Prédelouard en Aunis ${ }^{294}$, 33 ans à 75 l. par an, 40 l. d'av., nourri jusqu'à l'embarquement.

\section{8}

à Pierre Gaigneur, marchand pour la Compagnie des Indes Occidentales: (Teuleron, not. Liasse). [ 667 ] 6 avril - André Sire, journalier et couvreur d'ardoise, de Fontenay, 701.

[ 668 ] 11 avril - Louis Gouverir 295 , apothicaire, d'Apremont en BasPoitou ${ }^{296}, 60$ l. Signe.

[ 669 ] 2 mai - Isaac de Bellefaye, de Villegagnon, 751.

[ 670 ] 1er juin - Jean Damase, de Chef-Boutonne, $75 \mathrm{l}$.

[671] 4 juin - Louis Moreau, tailleur d'habits, de Pauléon en Aunis, 75 1. Signe.

[672a] 30 juin - Mathurin PelozEAU, journalier, de Chabournay en Poitou ${ }^{297}$.

[ 672b ] 7 juillet 1868 - Engagement de Bertrand de Leglebat, bonnetier à Toulouse, pour 3 ans à 80 livres par an, à Pierre
Gagneur qui agit au nom de la Compagnie des Indes Occidentales. Leglebat ira au Canada. Il signe (Teuleron not.)

1670

au sr Arnaud Péré, marchand à La Rochelle faisant pour les PP. Jesuites du Canada (Droayneau, not. Liasse).

[ 673 ] 21 mars - Etienne Ardouin, farinier, des Sables, pour 3 ans, à 100 l. 70 d'av. Signe.

[ 674 ] Philippe JARNY, farinier, des Sables, Signe.

Ils travailleront de leur profession.

au sr Alexandre Petit, marchand de cette ville, pour Québec, 3 ans (Drouyneau, not. Liasse). [ 675 ] 4 avril - Barthélémy HaUTIN, taillandier, d'Angers, travaillera de son métier pour $80 \mathrm{l}$. par an, "les outils et les materiaux nécessaires lui seront fournis". Promesse d'une avance de 501., si besoin.

à Daniel Biaille, marchand à La Rochelle, faisant pour Nicolas Gouvreau, arquebusier, demeurant à Québec (Drouyneau, not. Liasse).

[ 676 ] 12 avril - Bernard de LaNDABOURE, arquebusier, de Lahonce ${ }^{298}$, à une lieue de Bayonne, Mar.).

293. Commune du canton de Civray (Vienne).

294. Peut-être Puydrouard, commune de Forges, canton d'Aigrefeuille ( $\mathrm{Ch}$.

295. Ou Gonnerit.

296. Commune du canton de Palluau (Vendée).

297. Commune du canton de Vouillé (Vienne).

298. Commune du canton de Bayonne (Basses-Pyrénées). 
3 ans, comme arquebusier, à 1001. par an, 50 l. et les outils avancés.

\section{1}

Pour M. Talon, intendant à Québec' par Antoine Allaire, marchand à La Rochelle, par l'intermédiaire de Toussaint Quenet, marchand à Rouen (Teuleron, not. Liasse). [677] 4 mai - André Tubleau, 19 ans, de Luçon, 3 ans, 50 l. par an, 30 d'av.

à Arnaud Péré, marchand, pour M. L'évêque de Pétrée à Québec (Teuleron, not. Liasse).

[678] 18 mai - François GAY, laboureur, de Salle en Angoumois ${ }^{299}, 22$ ans, 3 ans, 60 l. par an, 40 l. d'av. nourri jusqu'à l'embarquement.

\section{3}

à Arnaud Péré, marchand, pour Québec, pour 3 ans (Michelon, not. Liasse).

[679] 13 avril - Guillaume LEFEBVRE, journalier, de SaintBrieuc,| 25 ans, à $80 \mathrm{l}$. par an, $40 \mathrm{l}$. d'av. et 6 sous par jour jusqu'à son embarquement.

[ 680 ] 14 avril - Martin Thomas,

- de Tours, 19 ans, à 60 1. par an,

31 l. d'av.

[681 ] Yves Martin, journalier, de Peaulle en Bretagne ${ }^{300}, 19$ ans, à 60 l. par an, 31 l. d'av.
[682] Jean Pasquereau, de La Rochelle, 20 ans, 60 1. dont 36 d'av. Il n'est pas question de nourriture jusqu'à son départ. Signe.

[683 ] 10 juin - Pierre SEurpe, tailleur d'habits, d'Hesdin, en Artois $^{301}, 17$ ans, 601 . dont 501 . 10 s. d'av. nourri jusqu'à l'embarquement. Signe.

à Jean Péré, marchand à La Rochelle, pour Québec, 3 ans (Michelon, not. Liasse).

[684] 28 avril - Pierre Brochet, journalier, de La Rochelle, 401. par an, 30 l. d'avance, 4 sous par jour jusqu'à l'embarquement.

[ 685 ] Henri Fromageau, journalier de La Rochelle, 40 l. par an, 30 l. d'avance, 4 sous par jour jusqu'à l'embarquement.

[ 686 ] 4 mai - François Couturier, journalier, de La Rochelle, $42 \mathrm{l}$. dont 30 d'av. 4 sous par jour jusqu'à l'embarquement. Signe. au même pour les PP. Jésuites de Québec "pour aller à Québec et autres lieux de la Nouvelle France" (même notaire).

[687] 9 juin - Jacques MarlloCHEAU, journalier, de Saint-Pouan en Poitou ${ }^{302}, 20$ ans, 3 ans, 751. 40 d'av.

à Michel Cressé, marchand et bourgeois de Paris, de présent à La Rochelle, prêt à partir pour Quebec (Drouyneau not. Liasse).

299. Il y a deux communes de ce nom en Charente, l'une canton de Ville Gagnon, l'autre du canton de Barbezieux.

300. Commune du canton de Maël-Carhaix (Côte du Nord).

301. Chef-lieu de canton du Pas-de-Calais.

302. Non identifié. 
[ 688 ] 4 juillet - Le sieur Philippe Rozer, marchand à La Rochelle, engage pour 3 ans qui commenceront ce même jour, son fils Etienne, âgé de 16 ans. Le père paie le voyage et donne $50 \mathrm{l}$. av. Michel Cressé qui est prêt à partir pour Québec. Ensuite son fils sera envoyé à la Guadeloupe.

\section{6}

à Jean Gitton, marchand à La Rochelle, pour le Canada "en le lieu qu'il plaira audit sieur Gitton" (Drouyneau, not. rég. 16761677 fol. $50 \mathrm{v}^{\circ}$ ).

[ 689 ] 13 mars Pierre Regnaud, 23 ans, de Saint-Georges de Chatelaison $^{303}$ en Anjou, pour 3 ans, à 75 1. par an, payées à la fin des 3 années.

à Messire François Lefebvre, prêtre, chapelain de la Chapelle de SaintNicolas des Noyers, paroisse Sainte-Marie-Madeleine d'Andilly en Normandie ${ }^{304}$, demeurant ordinairement au Séminaire Saint-Sulpice, logé à La Rochelle, (Drouyneau, not. Reg. 1676-1677, fol. $\left.111 \mathrm{v}^{\circ}\right)$.

[690] 13 mai - Philippe Bouyer, farinier, demeurant à La Rochelle, pour 3 ans, à 125 1. par an, 55 l. d'av. pour Montréal.

\section{8}

à Jean Gitton, pour 3 ans, (Reg. Drouyneau, 1678 fol. $58 \mathrm{v}^{\circ}$ ).

[691 ] 15 mars - Jacques GuiLLARMOT, tonnelier, de l'isle de Mascoult en Guyenne ${ }^{305}$, pour le Canada, 3 ans, travaillera de son métier à 120 l. par an, payées à la fin de chaque année, $40 \mathrm{l}$. d'av. aller et retour.

au même, pour le sieur Jacques Lebert, marchand à Montréal, pour 3 ans (Drouyneau, liasse). [692] 13 mars - Vincent JEAN, laboureur, d'Anjat en Saintong $\theta^{306}$ 75 l. dont 30 d'avance.

[693] 5 avril - Michel Leblond, menuisier, de Picardie, 1501. dont 60 d'av. Signe.

[694 ] 8 avril - Reçu de 30 sols par Vincent JEAN.

[695] 17 avril - Mathieu BorsFILs, laboureur, de l'île de Ré, 90 1. par an, 30 1. d'avance.

19 avril - Reçu de 51 . par le même. [696] 19 avril - Michel DoussaIN, farinier, de Nantes, 801. par an. (Reçu de 3 l. 15 s.).

au même pour le sr Richard Denis, marchand à Québec (Drouyneau not. liasse).

[697 ] 27 mars - Yvon RichaRD, marinier, de Saint-Gilles sur Vie ${ }^{307}$, pour 3 ans, à 170 l. par an, 801 . plus 15 l. d'avance.

303. Commune du canton de Doué-la-Fontaine (Maine-et-Loire).

304. Non identifié.

305. Hameau de la commune de Blanquefort (Gironde).

306. Soit Anjeac-Champagne, commune du canton de Segonzac, Charente, ou Anjeac-Charente, commune du canton de Châteauneuf (Charente).

307. Chef-lieu de canton de la Vendée. 
a Pierre Lagarde, marchand et habitant de Baussequand, pays de la Nouvelle France, pour 3 ans, à $60 \mathrm{l}$. du Canada par an et s'embarquera sur le Lion d'Or, cap. Monbœuf, actuellement à la Palice (Rabusson, not.)

[698] 6 avril - Eléon Deganne, laboureur à bœufs né à Marans, demeurant actuellement au bourg de Sainte-Soule en ce gouvernoment jouissant de ses droits, da consentement de Jacques Brochet, laboureur à bras, son oncle, à cause de Suzanne de Guiltre, sa femme, de Sainto-Soule.

1681

A Joseph Petit, sieur de Brumeau, marchand aux Trois-Rivières, actuellement à La Rochelle (Drouyneau, not. Liasse):

[ 699 ] 5 mai - Pierre Boudor, marchand demeurant à Limoges, met volontairement en servitude Jean, son fils âgé de 18 ans, qui sera fourni d'habit et dont le retour sera payé. L'engagement qui va jusqu'en octobre pourra être renouvelé un an aux mêmes conditions.

\section{3}

à Le Breton, de Québec, s'engage pour 3 ans (René Rivière not.)

[ 700 ] Jean Bertrand, laboureur, de Matha ${ }^{308}$.

aux PP. du Séminaire de Montréal, pour 3 ans (même not.)
[ 701 ] 19 avril - André Poudretr, bonnetier, de Poitiers, 24 ans, a 66 l. par an.

[ 702 ] 30 avril - Antoine de la FAYE, laboureur, de Poitiers, 501. par an. Signe.

[ 703 ] Hilaire Serrault, laboureur, de Vouzailles près Poitiers ${ }^{309}, 30$ ans, $75 \mathrm{l}$.

[ 704 ] Antoine Tessereau, laboureur, de Dissay, près Poitiers ${ }^{310}$, 23 ans, $75 \mathrm{l}$.

[705] 6 mai - Aimable Trouvé, laboureur, de la Guerche en Touraine ${ }^{311}, 18$ ans, 50 l. Signe.

\section{4}

à Jean Duret, capitaine du Samuel qui part pour le Canada (même not).

[ 706 ] 31 mai - François Gingros, 19 ans, menuisier, de La Rochelle autorisé par René Gingros, marchand, son curateur. Pour 3 ans, sans indication de gages.

\section{5}

au sr Moïse Moreau, marchand à La Rochelle, pour le marquis de Cheury et autres intéressés en la Compagnie d'Acadie, et partiront sur le Saint-Lourdes 200 tx. cap. Michel Caude, pour 3 ans, travailleront de leur profession, aller et retour (Berthelot, not. Liasse). [ 707 ] 7 mars - Antoine Tallon, marinier, de Marcilly en Aunis, à 110 l. par an, 30 l. d'avance.

308. Chef-lieu de canton de la Charente-Maritime.

309. Commune du canton de Mirebeau (Vienne).

310. Commune du canton de Saint-Georges (ibid.)

311. Commune du canton du Grand-Pressigny (Indre-\&-Loire). 
[ 708 ] Jacques Delamare, marinier, des Sables, à 110 1. par an, 301. d'avance.

[ 709 ] Olivier Laimable, marinier, du Havre, 100 l. en argent ou en marchandises.

[ 710 ] 8 mars Pierre Maran, tonnelier, de Marcilly, 751.

[ 711 ] 13 mars - Louis Grelat, de Moëze en Saintonge, aller et retour, 50 l. par an. Signe.

[712 ] Pierre Ayrault, tonnelier, de La Rochelle, pour 20 mois à 16 l. par mois, payées de six mois en six mois, 48 l. d'avance. Signe.

Aux Religieuses hospitalières de Montréal, s'engage pour 3 ans (Rivière et Soulard, not.)

[713] 21 mars - François GARNAUX, laboureur, de Saint-Jean d'Angély, 25 ans.

au sr Charles Aubert de la Chesnaye, de Québec (même not.).

[714] 27 avril - Jean Bounet, farinier, de La Rochelle.

[ 715 ] 7 mai - Louis Roy, de l'île de Ré.

[716] 7 mai - André de Mes, laboureur, de Beaumont près Pons ${ }^{312}$

[ 717 ] 14 mai - Honoré Pezeau, charretier, de Machecoul-en-Retz en Bretagne ${ }^{313}$.

\section{6}

à Antoine Héron, directeur de la Compagnie de l'Acadie (Rivière et Soulard, not.)

[ 718 ] 3 février - Jean Boutineau, pilote
[ 719 ] 4 février - Paul Chevalier, charpentier de navire.

[ 720 ] 6 février - Pierre Courtin, maître de Chaloupe.

[721 ] Pierre Giraud, maître de Chaloupe.

[ 722 ] Hélie Rapiée, maître de Chaloupe.

[ 723 ] Pierre Regnaud, Cassuin.

[ 724 ] Aaron Beuret, Cassuin.

[725] Samuel Gitteau, Cassuin.

[ 726 ] Jean RiorteaU, arimier.

[ 727 ] Jean Prudhomme, arimier.

[ 728 ] Pierre Mallet, arimier.

[ 729 ] Paul Chevalier, charpentier de navire.

[ 730 ] Pierre Courtin, arimier.

[ 731 ] 11 février - Joseph BeLot

[ 732 ] 12 février - Jean Courtin.

[733 ] 18 février - Jean JeantiNEAU.

[ 734] 20 février - Jean DesGourlloN, dit Vignot, chirurgien.

[ 735 ] 28 février - René ThibauDEAU, tonnelier.

[ 736 ] Daniel Savignen, tonnelier.

[ 737 ] 2 mars - Andrée Pinel, native de Québec.

[ 738 ] 6 mars - François Savarit, tailleur de pierre.

[ 739 ] 9 mars - Pierre GuYot, homme de service.

[ 740 ] 13 mars - Jacques LномMEAU, garçon marinier.

[741] Pierre Rochetead, garçon marinier.

[ 742 ] Jean Courtin, garçon marinier.

[743 ] Alain GarraIn, matelot.

à Gilles Boyvinet, lieutenant-gønఠral aux Trois-Rivières et direc-

312. Non identifié.

313. Chef-lieu de canton de l'arrondissement de Nantes (Loire-Inf.). 
teur du Domaine d'Occident au Canada, pour 3 ans (mêmes not.)

[ 744 ] 18 mai - Pierre Thomas, chirurgien.

[ 745 ] 10 août - Gaspard Pichon, garçon de service.

\section{7}

à Antoine Héron, directeur de la Compagnie d'Acadie, s'engage (Rivière et Soulard, not).

[ 746 ] 5 mars - Jean Adreint, garçon de service.

à Gabriel Gaultier, commis généra] de la Compagnie d'Acadie, s'engage en qualité de commis en Acadie (mêmes notaires).

[ 747 ] 10 mars - Germain Godin dit Beauregard, à 260 1. par an

à Soumande de Lorme, au Canada (mêmes notaires).

[748] 11 juin - Guillaume MESNIER, farinier.

[ 749 ] 20 juin - Jean Bonus.

[ 750 ] Philippe Guillemin, tanneur.

1688

Au service de la Compagnie d'Acadie (mêmes notaires).

[ 751 ] 29 mars - Jules Gillet.

[ 752 ] Pierre Tessier.

[ 753 ] 30 mars - René Maréchal.

[ 754 ] Gilles LeHÉdoIS.

à Lemoine d'Iberville, pour aller à la mer du Nord du Canada au service de la Compagnie dudit lieu (mêmes not.).
[ 755 ] 31 mars - Jacques VILLIERS, maître arquebusier.

[756 ] 3 avril - Michel PhilipPEAU, taillandier.

aux PP. Jésuites de Québec, pour 3 ans, à 75 l. de France, par an s'engagent pour labourer (Masson, not.)

[ 758 ] 24 juin - Jacques DelouCHE, 21 ans, fils de Damien Delouche, chirurgien-apothicaire en la paroisse de Buxière (?) au diocèse de Clermont ${ }^{314}$, et de Claudine Garnier. Signe.

[ 759 ] René Dubessay, fils de feu Jean Dubessay et de Jeanne Doulet, de Saint-Jean, au diocèse de Clermont ${ }^{315}$.

Jean Grignon, engage pour le $\mathrm{Ca}$ nada (Rivière et Soulard, not.)

[ 760 ] 30 juin - François RobIN, garçon de service.

[ 761 ] 2 juillet - Jean Sarrault.

1689

à Antoine Héron, agent de la Compagnie d'Acadie, pour aller servir sous les ordres de Laborde de Mainferme, cap. de l'Aventurier pendant le voyage de l'Acadie. (Rivière et Soulard not.)

[ 762 ] 22 mars - Claude MagnY. [ 763 ] Pierre Texier.

[ 764 ] Thomas Jouet.

[ 765 ] Mathurin Forget, tous matelots, des Sables d'Olonne.

à Jean Grignon, de La Rochelle, pour P. de Bétrancourt, écuyer, au Canada (mêmes not.).

314. Peut-être Buxières-sous-Montaigut, canton de Montaigut (Puy-de-Dôme)

315. Non identifié. 
[ 766 ] 18 avril - Gabriel Huppé. au même pour $M$. de Longueil, au Canada:

[767] 18 avril. Jean Nicolleau.

\section{1}

à Jean Grignon, marchand, pour aller servir 3 ans au Canada (Rivière et Soulard, not.).

[ 768 ] 12 mai - Jean Delasse, 25 ans, jardinier, de Bordeaux, 75 l. par an.

[ 769 ] 4 juillet - Louis Dalques, 40 ans, homme de service, de poil noir, de taille moyenne, $60 \mathrm{l}$. par an.

\section{2}

à MM. de la Compagnie du Nord du Canada, pour 3 ans (Rivière et Soulard not).

[ 770 ] 5 mai - François Brodu, 22 ans, garçon arquebusier, de la Chapelle-Paluan ${ }^{316}$ en Bas-Poitou, 300 l. de France par an, aller et retour payés.

1693

[ 771 ] 26 mars - Engagement de François Héliot, de Cancale ${ }^{317}$, pour Terre-Neuve (Rivière et Soulard).

\section{4}

à Pierre Lemoine d'Iberville, capitaine des frégates du roi, s'engage pour 3 ans (Rivière et Soulard, not.).
[772] 14 mai - Gilles Lord, 21 ans, garçon taillandier de Toulouse à 160 1. par an.

à Jean Grignon, marchand à La Rochelle, s'engage pour 3 ans pour faire la pêcherie et le séchage de la morue (même not.).

[773] Joannis Berasteguy, 42 ans, de Saint-Jean de Luz ${ }^{318}$, maître de grave, à 500 l. par an. Signe.

1695

à Jean Jung de Saint-Laurent, marchand de Bordeaux, pour 3 ans, passant sur le Saint Louis aller le servir 3 ans au Canada $90 \mathrm{l}$. de France en tout, et en renonçant aux bénéfices de la Déclaration du roi (Guillemot, notaire). [ 774 ] 24 juin - René de la Lande, 18 ans, d'Ambroise,

1696

à Jacques Leber, de Montréal, représenté par Jacques Leber, son fils, pour 3 ans à $80 \mathrm{l}$. du Canada, par an: (Rivière et Soulard not.). [ 775 ] 1er mai - Pierre Resneau, 38 ans, de La Rochelle.

à Jean Paré, marchand de La Rochelle, pour Aubert de la Chesnaye à Québec, pour 3 ans (Rivière et Soulard not.).

[776 ] 15 juin - Vincent Arnou, 21 ans, garçon de service, de la ville de Mirebeau en Poitou, qui signe, 140 l. par an.

316. Commune du canton des Sables d'Olonne (Vendée).

317. Chef-lieu de canton de l'Ille-et-Vilaine.

318. Chef-lieu de canton des Basses-Pyrénées. 
[ 777 ] Joseph Aubert, 22 ans, id. garçon de service, de la ville de Mirebeau en Poitou, qui signe, 140 1. par an.

à P. Lemoine d'Iberville, pour servir sa femme ${ }^{319}$ (Rivière à Soulard not.).

[ 778 ] 26 novembre - Elisabeth LAFond, 36 ans, de Bordeaux, 3 ans à 60 1. par an.

\section{8}

à Jacques Pacquinet et à Abraham Aubier, maître et constructeur de navires, s'engagent pour l'Acadie (Bagard, not.).

[ 779 ] 4 avril - Jacques Turpaud, chirurgien, des Sables d'Olonne.

[ 780 ] Alexandre de Vézin, scieur de long, de La Rochelle

[781 ] Simon CoIndet, scieur de long, de La Rochelle.

à Charles Lemoine, écuyer, sr de Longueil, capitaine d'une compagnie de la marine au Canada et à dame Elisabeth Souärt s'engage pour 3 ans, comme garçon de service (Rivière et Soulard, not.).

[782] 9 juin - Nicolas MAUdINIÈRE, 25 ans, maçon de profession de Concré au Maine ${ }^{320}, 601$. par an.

à Pierre Lesueur, bourgeois et marchand à Montréal, pour 3 ans s'engagent pour servir au Canada et même au pays de Mississipi (Rivière et Soulard, not.).

[ 783 [ 14 juin - Yves Pinet, 22 ans, garçon arquebusier, à La Rochelle à 200 1. par an. Signe.

[784] Henri Picauron, 21 ans, garçon taillandier, de Moscoup ${ }^{321}$ (?) 100 l. par an.

à Joseph Le Goué, sieur de Grays, pour 2 ans (Bagard, not.).

[ 785 ] 15 juin - Antoine Bouton, taillandier, de Saint-Jean, à sept lieues de Lyon $^{322}$. Le maître fournira le charbon, mais les profits seront partagés.

à M. de Callières, gouverneur de Montréal (même notaire)

[ 786 ] 16 juin - Pierre Pravial, cuisinier, de Toulouse, pour 3 ans, à 120 l. par an.

\section{9}

à Pierre Martel, marchand à Québec, pour 5 ans, à $150 \mathrm{l}$. par an (Rivière et Soulard, not.).

[ 787 ] $20 \mathrm{mai}$ - Jean SonaIluon, 26 ans, garçon boulanger, de Marmande ${ }^{323}$, qui signe.

au sr Charles Pauperet, marchand à Québec, pour 3 ans, à 601 . de France par an (mêmes notaires). [ 788 ] 12 juin - Côme Gilbert, 23 ans, garçon de service, d'Orléans, Signe.

319. Marie-Thérèse Pollet de la Courbe Pocatière (Cf. 'E. de Cathelineau,

"Les deux beaux mariages d'une Canadienne", in Nova Francia, (1931): 114-186.

320. Commune du canton de Montfort (Sarthe).

321. Non identifié.

322. Sans doute Saint-Jean-de-Bournay (Isère).

323. Lot-et-Garonne. 
à la dame veuve Pachot, de Québec, pour 3 ans, à 250 livres (mêmes not.).

[ 789 ] 30 juin - Le sieur Antoine LAgarde, 23 ans, de Montauban $^{324}$. Signe.

au sr Jean Peyre "sans prétention d'aulcunes choses de part et d'autre" (id.).

[ 790 ] 1er juillet - Bernard $\mathrm{CA}_{A-}$ PELA, 22 ans, garçon de service, de Saint-Nicolas de la Grave en Lauguedoc ${ }^{325}$.

1700

à messire Soumande, prêtre, chanoine de la cathédrale de Québec, s'engagent pour trois ans, tous comme laboureurs à $50 \mathrm{l}$. par an (même not.).

[ 791 ] 24 mai - Nicolas Jouanne, 23 ans, de Briquebec, en Basse Normandi $^{326}$.

[ 792 ] Pierre Gautier, 23 ans, de Saint-Sulpice en Périgord ${ }^{327}$.

[ 793 ] Théodore MouLin, 25 ans, de Kildern, près Dublin en Irlande.

[ 794 ] Etienne Préau, 19 ans, de Blaye.

[ 795 ] Jean Mouroux, 20 ans, du Berry.

à Jean Lagrange, du Canada, pour 3 ans, à 50 l. apr an (mêmes not.).
[796] 15 juin - Jean Bouclier, 21 ans, garçon de service, d'Autrat (?) près Barbézieux ${ }^{328}$.

\section{2}

A MM. du Séminaire de Québec, pour 3 ans, à $120 \mathrm{l}$. par an (mêmes not).

[797] 13 mars - François $\mathrm{BA}_{\mathrm{A}}$ REAU, 22 ans, farinier à moulin à eau, de Bournezeau ${ }^{329}$ en Poitou, demeurant à La Rochelle.

\section{4}

à Louis Audet et à Pierre Cos, écuyer, sieur de Bailleul, enseignes d'une compagnie de la marine (mêmes notaires).

[ 798 ] 9 juillet - Pierre Chevrier, 22 ans, de Rennes, pour 3 ans à 36 l. par an, comme domestique.

1706

au Marquis de Vaudreuil, gouverneur du Canada, absent, pour 3 ans à 100 l. de France par an (mêmes notaires).

[ 799 ] 4 mai - Etienne Grandpré, 24 ans, garçon boulanger de Montpezat près d'Agen ${ }^{330}$. Signe.

1708

à Pierre Plassan, marchand à Quebec, (mêmes notaires).

324. Tarn-et-Garonne.

325. Chef-lieu de canton du Tarn-et-Garonne.

326. Chef-lieu de canton, Manche.

327. Sans doute Saint-Sulpice d'Exideuil, canton de Lanouaille (Dordogne). 328. Non identifié.

329. Commune du canton de Chantonnay (Vendée).

330. Chef-lieu de canton du Tarn-et-Garonne. 
[ 800 ] 1er juillet - le sr Charles Gontault, 24 ans, de Blaye, 3 ans 300 l. de France par an. Signe. [801] 6 juillet - Jacques DeLAVAL, 15 ans, garçon de service, de Châlons en Champagne ${ }^{331}, 4$ ans, avec le consentement de son père Claude Delaval. Il n'aura que son entretien.

[ 802 ] 13 juillet - René Foucauld, 14 ans, fils de feu Denis Foucauld maître canonnier au port de Rochefort et de Marguerite Martin, pour 4 ans.

\section{4}

a Pierre You, sr de la Découverte, aide-major à Montréal (même not).

[ 803 ] 12 janvier - François JANIX, 19 ans, de Jarnac ${ }^{332}$, pour 5 ans, à $100 \mathrm{l}$. pour les 5 ans.

[ 804 ] 23 avril - Jacques Bongard, 21 ans, de Chasmouillet, paroisse de Dompierre ${ }^{333}$.

à Claude de Ramsay, sr de la Gesse, Montigny et Bois-Fleurance, gouverneur de Montréal, pour 5 ans à 60 l. par an (mêmes not.).

[ 805 ] 8 avril - Philippe Texier, 25 ans, d'Avrillé en Poitou ${ }^{334}$.

[ 806 ] 9 avril - Pierre Delafres-
NAYE, 16 ans, de Lunéville en Lorraine $^{335}$.

[807] 12 avril - Jean Chasseu, 47 ans, de Cressé, près Beauvoir en Saintonge ${ }^{336}$.

[808] 14 avril - Claude MAUPoINT, 31 ans, de la Charite-surLoire $^{337}$.

aux sœurs de la Congrégation à Montréal 3 ans à $50 \mathrm{l}$. par an (mêmes notaires).

[ 809 ] 21 avril - Pierre MaIn, 22 ans, de Saint-Martin d'Entraigues près Chef-Boutonne ${ }^{338}$.

à Martin Delino, au Canada (id.). [810] 24 avril - Elie Lafarge, 26 ans, de Cadillac, comme ${ }^{339}$ domestique.

à Pierre Delestage Cie marchands au Canada, 3 ans. (id.)

[ 811 ] 24 avril - Toussaint FAUsse, 24 ans, domestique, de Bordeaux, 751 .

[812] 6 mai - Charles Daniel, tonnelier, de La Rochelle, 1201. par an.

à Longueuil, lieutenant de roi à Montréal, 3 ans, à 150 l. par an (mêmes notaires).

[ 818 ] 6 mai - Jean Mousseau. 25 ans, farinier, d'Aigrefeuille,

331. Châlons-sur-Marne (Marne).

332. Chef-lieu de canton de la Charente.

333. Commune du canton Est de La Rochelle.

334. Commune du canton de Talmont (Vendée).

335. Meurthe-et-Moselle.

336. Beauvoir, chef-lieu du canton des Deux-Sèvres.

337. Chef-lieu de canton de la Nièvre.

338. Commune du canton de Chef-Boutonne (Deux-Sèvres).

339. Il est deux Cadillac dans la Gironde. 
[ 814 ] François Bouveron, gargon tonnelier, de La Rochelle.

à La Framboise, marchand aux Trois-Rivières (mêmes notaires)

[815] Louis Devau, 21 ans, de. Saint-Laurent en Aunis ${ }^{340}, 3$ ans à $150 \mathrm{l}$. par an.

[ 816 ] 29 mai - Jean Dellaunay, laboureur, de la Madeleine, d'Angers.

à Juchereau du Chesnay sr de Beauport, au Canada, s'engagent (mêmes notaires).

[ 817 ] 1er avril - Julien Lespinay dit Saint-Paul, homme de service de Hirel, évêché de $\mathrm{Dol}^{341}$

à Bernard Veyres, au Canada, (mêmes notaires).

[818] 20 mai - Jean ToussainT, retailleur en roues, de Civray en Poitou.

[ 819 ] Jean Raballet, garçon de service, du Mont-Dore en Périgord $^{342}$.

[ 820 ] François Achendeau, laboureur, du Poitou.

à Dujay, pour Québec, (mêmes notaires).

[ 821 \}. 11 juin - Joseph Benoist, chirurgien, de Ferrières, ${ }^{343}$ province de Sens.

[ 822 ] 12 juin - Jean-Louis GiverNEAU, vitrier, de Saint-Macaire en Gascogne ${ }^{344}$.
[823] Joseph Fremern, tailleur, de Saint-Laurent de Paris.

[824] 18 juin - Joseph Texier, farinier, de la paroisse du Val, ఠิêché du Mans ${ }^{345}$.

à Sarrasin, conseiller au conseil souverain de Québec (mêmes notaires).

[ 825 ] 30 avril - ..... SARreau, garçon de service, de Marennes [ 826 ] 13 mai - François BARANGER, laboureur.

[ 827 ] Jacques Buron, 38 ans, laboureur, de Tours.

à Jean Quenet, marchand à Montréal (mêmes notaires).

[ 828 ] 30 avril - Mathurin OlleREAU, laboureur, de Dompierre.

à Jean Dametzague, de Montréal (mêmes notaires).

[829] 7 mai - Pierre Massé, de la Fond, paroisse de N.D. de La Rochelle.

[ 830 ] 17 mai - Jean Dreilllard, de l'île de Ré.

[ 831 ] Jean Lucas, de la CroixChapeau ${ }^{346}$.

à Guillaume Dubois, cap du SaintMichel, pour le servir au Canada (mêmes not.).

[ 832 ] 25 juin - Pierre Arnaudin, bonnetier, de Blaye.

[ 833 ] Pierre Bouyer, escardeur de laine, de Cherves en Angoumois ${ }^{347}$.

340. Saint-Laurent-de-la-Prée, commune de la Charente-Maritime.

341. Commune du canton de Cancale (Ille-et-Vilaine).

342. Commune du canton de Rochefort (Puy-de-Dôme).

343. Peut-être Ferrières, hameau de la commune d'Andrys (Vienne).

344. Chef-lieu de canton de la Gironde.

345. Commune de la Sarthe.

346. Commune du canton de La Jarrie (Charente-Maritime).

347. Il est deux Cherves en Charente. 